\title{
ALKBH5 regulates somatic cell reprogramming in a phase specific manner
}

\section{Sherif Khodeer $^{1^{*}}$, Arne Klungland ${ }^{1,2}$, John Arne Dahl ${ }^{1 *}$}

1 Department of Microbiology, Oslo University Hospital, Rikshospitalet, Forskningsveien 1, 0373.Oslo, Norway.

${ }^{2}$ Department of Biosciences, Faculty of Mathematics and Natural Sciences, University of Oslo, 0316.Oslo, Norway.

\section{*Correspondence}

Sherif Khodeer and John Arne Dahl

Department of Microbiology, Oslo University Hospital, Rikshospitalet, Forskningsveien 1, 0373. Oslo, Norway.

Emails: Sherif.Khodeer@rr-research.no , j.a.dahl@medisin.uio.no

Keywords: Alkbh5, reprogramming, induced pluripotent stem cells (iPSCs), Nanog.

Running Title: ALKBH5 in somatic cell reprogramming 


\section{Author contributions:}

S.K. and J.A.D. discussed and designed the study. S.K designed all experiments. S.K performed the experiments and analyzed the data. J.A.D. supervised the work. A.K. supervised mouse mating and contributed the mice. S.K wrote the first draft of the manuscript and J.A.D revised and edited it. A.K. read and commented on the manuscript. All authors read and approved the final manuscript.

\section{Disclosure of potential conflicts of interest}

The authors declare they have no potential conflicts of interest.

\section{Acknowledgments}

The authors thank the Norwegian Transgenic Center (Norsk Transgen Senter (NTS)) and in particular; Shiasta Khan, Ingunn Jermstad, and Dr. Knut Tomas Dalen for setting up and maintaining floxed Alkbh5 mice mating, Guro Flor Lien and Gaute Nesse for maintaining and genotyping knockout Alkbh5 mice. This work was supported by the South-Eastern Norway Regional Health Authority Grant 2018063, and by the Research Council of Norway, Young Research Talents Grant 289467 (to John Arne Dahl). 


\begin{abstract}
Establishment of the pluripotency regulatory network in somatic cells by introducing four transcriptional factors, (octamer binding transcription factor 4 (OCT4), SRY (sex determining region Y)-box 2 (SOX2), Kruppel - like factor 4 (KLF4), and cellular-Myelocytomatosis (cMYC) provides a promising tool for cell-based therapies in regenerative medicine. Still, the mechanisms at play when generating induced pluripotent stem cells from somatic cells is only partly understood. Here we show that the RNA specific N6-methyladenosine $\left(\mathrm{m}^{6} \mathrm{~A}\right)$ demethylase ALKBH5 regulates somatic cell reprogramming in a stage specific manner. Knockdown or knockout of Alkbh5 in the early reprogramming phase impairs the reprogramming efficiency by reducing the proliferation rate through arresting the cells at G2/M phase and decreasing the mesenchymal-to-epithelial transition (MET) rate. However, there is no significant change in reprogramming efficiency when Alkbh5 is depleted at the late phase of reprogramming. On the other hand, ALKBH5 overexpression at the earlyreprogramming phase has no significant impact on reprogramming efficiency, while overexpression at the late phase enhances the reprogramming by stabilizing Nanog transcripts resulting in upregulated Nanog expression. Our study provides mechanistic insight into the crucial dynamic role of ALKBH5 in regulating somatic cell reprogramming at the posttranscriptional level.
\end{abstract}




\section{Introduction}

The four transcriptions factors OCT4, SOX2, KLF4, and c-MYC (OSKM) are sufficient to reprogram and induce pluripotency when ectopically expressed in mouse or human somatic cells, to generate induced pluripotent stem cells (iPSCs) $[1,2]$. These reprogrammed iPSCs are highly similar to their pluripotent embryonic stem cell (ESC) counterparts in transcriptional profile and epigenetic landscape [3-5], and show infinite selfrenewal capability [2], and the ability to differentiate to the three germ layers in vivo and in vitro [6]. Therefore, iPSC technology provide an ideal tool for drug screening, patient-specific disease modeling, and hold great promise for therapeutic applications in the future [7].

The early phase of the reprogramming process is characterized by stochastic events [8] , in which the mesenchymal genes are downregulated, while epithelial genes are upregulated in a process known as mesenchymal-epithelial transition (MET), together with clear morphological transformation accompanied with increased proliferation rate to form cellclusters $[9,10]$. Despite that, most fibroblasts exposed to iPSC reprogramming conditions fail to achieve the proper morphological changes and remain in a fibroblast like morphology. These trapped cells undergo senescence, apoptosis, and cell cycle arrest, which in turn explain the low efficiency of the reprogramming process [11-13]. Besides, several studies have demonstrated that cell cycle regulators including $\mathrm{p} 21$, p53 or p16/INK4A are barriers to the reprogramming process and their depletion enhances the reprogramming process [14-17].

The late phase of the reprogramming process is considered deterministic, in which reactivation of endogenous Sox 2 expression is considered a rate-limiting step for acquiring the ESCs identity [8] .This phase is also characterized by removal of somatic epigenetic memory, telomere elongation, expression of endogenous pluripotency genes, and establishment of pluripotency specific epigenetic and transcriptional profiles $[9,10]$. 
The N6-methyladenosine $\left(\mathrm{m}^{6} \mathrm{~A}\right)$ modification, methylation of the N6 position of the adenosine base, is the most abundant internal posttranscriptional modification in mammalian mRNA [18]. It was recently showed that $\mathrm{m}^{6} \mathrm{~A}$ modification is reversible and its presence is regulated through coordination of several modulators $[19,20]$. Positioning of $\mathrm{m}^{6} \mathrm{~A}$ is mediated by methyl transferase-like 3 (METLL3), methyl transferase-like 14 (METLL14) and Wilms' tumor 1-associating protein (WTAP) [21-24]. Removal of $\mathrm{m}^{6} \mathrm{~A}$ is carried out by the demethylases fat mass and obesity-associated protein (FTO) and alkylated DNA repair protein AlkB homolog 5 (ALKBH5) [20, 25]. Furthermore, the $\mathrm{m}^{6} \mathrm{~A}$ modification is recognized and bound by readers including YTH domain-containing proteins 1-3 (YTHDF1-3) and (YTHDC1 and 2) which in turn facilitate downstream processing such as mRNA splicing, stabilization, translation or degradation [26-28].

ALKBH5 is one of nine mammalian members of the AlkB family of Fe (II) and $\alpha$ ketoglutarate dependent dioxygenases, and can demethylate the $\mathrm{m}^{6} \mathrm{~A}$ modification in RNA to adenosine (A) [24]. We have previously shown that Alkbh5 is highly expressed in meiotic cells of the testis and is mainly localized to the nucleus [24]. ALKBH5 has been shown to regulate various biological and pathophysiological processes including; autophagy, glioblastoma, breast cancer, lung cancer and infertility [24, 28-32]. In addition, the heterogeneity in Alkbh5 expression in several cancer models has led to suggestions of a putative oncogenic or tumor suppressive role [33]. Despite extensive studies on ALKBH5 in different biological systems, the functional and regulatory role of ALKBH5 in somatic cell reprogramming has not been addressed. In this study, we dissected the precise role of ALKBH5 in the reprogramming process and our data revealed that ALKBH5 exhibits a biphasic role during somatic cell reprogramming. Depletion of Alkbh5 in the very early phase of reprogramming impairs the reprogramming process through downregulation of Cyclin B1 and $\mathrm{B} 2$ resulting in reduction in the cell proliferation rate, and arresting cells at G2/M phase 
accompanied by decreasing the rate of MET. In the late phase, overexpression of Alkbh5 stabilizes Nanog transcripts resulting in upregulated Nanog expression, which in turn enhances the reprogramming efficiency.

\section{Results}

\section{ALKBH5 depletion in early phase impairs reprogramming efficiency}

To explore the role of ALKBH5 in reprogramming, we first examined the expression of Alkbh5 during the reprogramming process, and we found that the expression of ALKBH5 is gradually upregulated during reprogramming at both the mRNA and protein levels (Fig. 1A, B). Then we used two different short hairpin RNAs (shRNA) to knockdown Alkbh5 expression (Fig. 1C). As expected by knocking down Alkbh5, we found that the total $\mathrm{m}^{6} \mathrm{~A}$ level at mRNA was highly increased compared to the controls (supplementary Fig.1A)

Next, we sat up a reprogramming system where Alkbh5 is knocked down 2 days before induction of retroviral reprogramming factors (OSKM) in mouse embryonic fibroblasts (MEFs), and we assessed the reprogramming efficiency on day 7 by flow cytometry and on day 14 by alkaline phosphatase (ALP) staining (Fig. 1D). Interestingly, Alkbh5 knockdown significantly reduced the reprogramming efficiency by decreasing the percentage of stagespecific embryonic antigen1 (SSEA1) positive cells (an early reprogramming marker) on day 7, and the number of ALP positive colonies on day 14, as compared to controls (Fig. 1E, F). To substantiate these data, we derived of Alkbh5 knockout (KO) MEFs and we found the reprogramming efficiency of Alkbh5 (KO) MEFs is greatly reduced compared to wild type (WT) MEFs either on day 7 or day 14 by decreasing the percentage of SSEA1 positive cells in the population (Fig. 1G and supplementary Fig.1B, C) [24]. Taken together, these data 
suggests that Alkbh5 depletion at the early phase of reprogramming impairs somatic cell reprogramming.

To further characterize the time specific role of ALKBH5, we took advantage of adoxycycline (DOX) inducible short hairpin RNA (shRNA) expression to suppress the expression of Alkbh5 at specific time points during reprogramming (Supplementary Fig. 1 D). We found that Alkbh5 knockdown at the very early stage of reprogramming, earlier than day 3, has the largest impact on reducing the reprogramming efficiency as shown by decreased fraction of SSEA1 positive cells on day 7 and 14 of reprogramming (Supplementary Fig. 1, E). On the other hand, we did not see any significant change in reprogramming efficiency when Alkbh5 was knocked down specifically at a later time than day 3 of the reprogramming process (Supplementary Fig. 1 E, F). Furthermore, we derived homozygous floxed Alkbh5 $($ Alkbh5 f/f $)$ MEFs and we used a 4-hydroxy tamoxifen (4-OH Tam) inducible Cre recombinase system, in which Cre is flanked by mutated ligand-binding domains of the murine estrogen receptor (Mer-Cre-Mer), to deplete Alkbh5 at specific time points during reprogramming (Supplementary Fig. 1 G-I) [24]. Consistent with our time specific knockdown data, depletion of Alkbh5 only at the very early stage (day 2) of reprogramming impairs the reprogramming as measured by a decreased percentage of SSEA1 positive cells in the population (Fig. $1 \mathrm{H}$ ). Time specific depletion of $A l k b h 5$ at day 8 or 10 of reprogramming has no significant impact on the reprogramming efficiency (Supplementary Fig. $1 \mathrm{~J}$ ). We further confirmed our data by treating homozygous floxed Alkbh5 MEFs with (4-OH Tam) to deplete Alkbh5 at different time points of reprogramming, and we found that only Alkbh5 depletion on day 2 or day 4 has a major impact on reducing the reprogramming efficiency as measured by alkaline phosphates staining at day 14 (Supplementary Fig. 1 K). In conclusion, only Alkbh5 depletion at the very early stage of reprogramming negatively affects the reprogramming process. 


\section{Effect of Alkbh5 removal during the early phase of reprogramming on cell cycle regulators and MET}

To investigate the mechanism involved in reduced reprogramming efficiency resulting from loss of Alkbh5, we focused on two important events; cell proliferation and MET that have both been reported to be critical to the early phase of reprogramming $[9,10]$. First, we explored the impact of Alkbh5 removal on proliferation and apoptosis during the early phase of reprogramming. Our data revealed that Alkbh5 knockdown during the early phase of reprogramming increases the percentage of cells at G2/M phase (Fig. 2A, B). Additionally, Alkbh5 depletion resulted in reduced cell proliferation (Fig. 2C). However, we did not see any significant changes in the percentage of Annexin positive cells as compared to the control, indicating that the reduction in cell number is mainly due to $\mathrm{G} 2 / \mathrm{M}$ cell cycle arrest (Supplementary Fig. 2A-C). Next, we assessed the expression of factors of the mitotic checkpoint complex (MCC) and found that Cyclin B1 and B2 are markedly downregulated at both the RNA and protein level after knocking down Alkbh5 during the early phase of reprogramming (Fig. 2D, E). Other MCC factors such as Cdc20, Mad1, Mad2, Bubl and Bub3 or G1 phase cell cycle regulators such as p16 and p19 were not significantly affected (Fig. 2D, E and supplementary Fig. 2D). To validate our Alkbh5 knockdown data, we used $A l k b h 5^{f f f}$ MEFs and induced Alkbh5 removal by (4-OH Tam) just 8 hours after reprogramming induction. In agreement with our knockdown data, we found reduction in Cyclin B1 and B2 levels showing that this phenotype is present with the loss of Alkbh5 both in MEFs and in the early reprogramming process (Supplementary Fig. 2E). It is also noteworthy that depletion of Alkbh5 in MEFs decreased the proliferation rate, and induced cell cycle arrest at G2/M phase accompanied by reduction in the protein level of both Cyclin B1 and B2 (Supplementary Fig. $2 \mathrm{~F}-\mathrm{I}$ ). This is consistent with what we observed during reprogramming (Fig. 2A -E). Thereafter, we assessed the MET process at day 6 of reprogramming in which Alkbh5was 
knocked down 2 days before reprogramming induction. Our qPCR and western blot data revealed that Alkbh5 depletion impair the MET process by decreasing the rate of downregulation of mesenchymal markers such as Platelet Derived Growth Factor Receptor Beta (PDGFR $\beta$ ), Snail Family Zinc Finger 2 (Slug), Zinc Finger E-Box Binding Homeobox 1 (Zeb1) and Zinc Finger E-Box Binding Homeobox 2 (Zeb2), and upregulation epithelial markers such as E-cadherin (E-cad), Epithelial Cell Adhesion Molecule (Epcam), and Occludin (Fig. 2F, G). Tamoxifen induced deletion of Alkbh5 eight hours after reprogramming supported this role of ALKBH5 in the MET process during reprogramming (Supplementary Fig. 2J). The role of ALKBH5 in MET is further supported by our observations on by tracking morphological changes during reprogramming after Alkbh5 depletion (Supplementary Fig. 2K). In summary, Alkbh5 is required for proper cell proliferation and for proper MET in the early phase of reprogramming.

\section{ALKBH5 overexpression in the late phase enhances reprogramming efficiency by upregulating Nanog}

We assessed the impact of ALKBH5 overexpression on the reprogramming process. Our data revealed that, overexpression of either ALKBH5, or ALKBH5 with a carboxyl terminal hemagglutinin (HA) tag (ALKBH5-HA), enhance the reprogramming process as measured by an increase in the percentage of SSEA1 positive cells and increase in number of ALP positive colonies at day 14 of reprogramming (Fig. 3A-C).

To investigate at what time of the reprogramming that ALKBH5 overexpression enhances reprogramming efficiency, we used a dox inducible overexpression system. We did not find any significant effect of ALKBH5 overexpression on the reprogramming efficiency at the early phase from day 1 to day 7 . However, the percentage of SSEA1 positive cells at day 14 is greatly increased after overexpression of ALKBH5-HA from day 1-14, as well as 
after overexpression from day 7-14 only (Fig. 3D). To investigate the molecular mechanism responsible for enhancing reprogramming efficiency by overexpression of ALKBH5 at the late phase, we used a dox inducible system for temporal overexpression of ALKBH5 from day 10 to day 12 (Fig.4 A). We found that overexpression of ALKBH5 result in upregulation of the endogenous RNA level of reprogramming factors such as Oct4, Sox2 and Klf4, and also other pluripotency factors including Klf2, Tbx3, Rexl, Esrrb, and in particular Nanog (Fig. 4B, C). We obtained similar results by overexpression of ALKBH5 from day 8 to day 10 (Supplementary Fig. 4 A-C). Previous studies have reported that Nanog is regulated postranscriptionally in both mouse and human ESCs by the $\mathrm{m}^{6} \mathrm{~A}$ machinery $[34,35]$. We hypothesized that Nanog transcripts are posttranscriptionally regulated through the $\mathrm{m}^{6} \mathrm{~A}$ modification during reprogramming and that overexpression of the $\mathrm{m}^{6} \mathrm{~A}$ demethylases ALKBH5 will reduce $\mathrm{m}^{6} \mathrm{~A}$ levels, potentially affecting the stability of Nanog transcripts. To test this hypothesis in the reprogramming context, we did $\mathrm{m}^{6} \mathrm{~A}$ IP at day 12 of reprogramming and indeed found that overexpression of ALKBH5 decreases the $\mathrm{m}^{6} \mathrm{~A}$ level at Nanog transcripts (Fig.4D). Then by checking the stability of Nanog transcript after overexpression of ALKBH5, we found that overexpression of ALKBH5 increases the stability of Nanog transcripts (Fig.4E). Finally, we assessed whether ALKBH5 overexpression could rescue the Alkbh5 KO phenotype in reprogramming. Our data revealed that overexpressing either ALKBH5 or ALKBH5-HA in Alkbh5 KO MEFs could restore the reprogramming efficiency (Fig.4F supplementary Fig. D-F). Taken together, our findings suggest that ALKBH5 overexpression in the late phase of reprogramming enhances the reprogramming efficiency by decreasing the $\mathrm{m}^{6} \mathrm{~A}$ level at Nanog transcripts, thus stabilizing these transcripts resulting in upregulation of Nanog. 


\section{Discussion}

Ectopic expression of the four transcription factors OCT4, SOX2, KLF4, and c-MYC in somatic cells can establish the pluripotency regulatory circuitry, resulting in massive changes at both the epigenetic and transcriptional level and the generation of iPSCs $[1,2]$. Successful therapeutic application of these iPSCs will likely require a comprehensive understanding of the molecular mechanism underlying somatic cell reprogramming. Here, we aimed to dissect the role of the $\mathrm{m}^{6} \mathrm{~A}$ demethylases ALKBH5 in somatic cell reprogramming.

Resetting the pluripotency cell cycle pattern is an essential step of achieving successful iPSC generation, suggesting cell division rate is a key parameter for somatic cell reprogramming [36]. In agreement with that, p53 and Ink4/Arf have been shown to act asbarriers to the reprogramming process [14-16]. Additionally, G2/M cell cycle regulators have been reported in maintaining pluripotency and the Cdk1/Cyclin B1 complex has been reported in enhancing the reprogramming process $[37,38]$. Moreover, the $\mathrm{m}^{6} \mathrm{~A}$ machinery has been reported to be involved in regulating Cdk1 and Cyclin B2, and knockout of Fat mass and obesity-associated (Fto) results in decreased expression of Cdk1 and Cyclin B2 causing G2/M cell cycle arrest in spermatogonia [39]. Here, we showed that Alkbh5 depletion in MEFs or during early phase of somatic cell reprogramming decreased the expression of Cyclin B1 and B2 accompanied by cell cycle arrest at G2/M phase, which in turn resulted in reduced proliferation and MET transformation rate, ultimately leading to impaired reprogramming efficiency. We did not formally exclude the possibility that Alkbh5 might have a direct effect on MET, which would be an interesting point for future studies. Furthermore, in contrast with that observed for the early phase of reprogramming, we found that depletion of Alkbh5 in the late phase of reprogramming did not have a significant effect on reprogramming efficiency. This indicates that the negative effect Alkbh5 depletion has on reprogramming efficiency 
plays out specifically during the early phase where both the resetting of the cell cycle pattern and morphological transformation to epithelial like cells occur.

Recent studies have revealed that the $\mathrm{m}^{6} \mathrm{~A}$ modification on mRNA is essential in regulating pluripotency and self-renewal of stem cell, somatic cell reprogramming and early embryonic development [28-31]. Regulation of pluripotency by $\mathrm{m}^{6} \mathrm{~A}$ has been reported in both mouse and human ESCs where Mettl3 and/or Mettl14 depletion induce a hyperpluripotent state presumably through increasing the $\mathrm{m}^{6} \mathrm{~A}$ level over several pluripotency related transcripts such as Nanog, resulting in increased transcript stability that hinder cells to exit from the pluripotency state $[34,35]$. NANOG is a key regulator of pluripotency and is required for acquiring pluripotency during the late phase of reprogramming [40, 41]. A synergistic role of Nanog in overexpression together with DNA demethylating agents in the late phase of reprogramming has been reported to enhance acquisition of the pluripotency state $[41,42]$. Moreover, NANOG co-binds with OCT4, SOX2 and KLF4 to many regulatory regions to facilitate binding of co-activator P300 [43]. Here we showed that ALKBH5 overexpression in the late phase of reprogramming decreases the $\mathrm{m}^{6} \mathrm{~A}$ level at Nanog transcripts, resulting in increased Nanog stability leading to enhanced reprogramming efficiency. Consistent with our findings, ALKBH5 has been reported to positively regulate Nanog stability and expression in response to hypoxia-inducible factor (HIF)- $1 \alpha$ - and HIF- $2 \alpha$ in breast cancer stem cells (BCSCs) [31].

A recent study reported that YTHDF2/3, but not YTHDF1, regulate the MET event in somatic cell reprogramming in an $\mathrm{m}^{6} \mathrm{~A}$ dependent manner through the Hippo signaling pathway effector Tead2 [44]. Other studies have shown redundancy among the three paralogs Ythdf1/2/3, suggesting they can have adequate functional compensation at least in some biological contexts $[45,46]$. It would be interesting to assess the role of $Y t d h f 1 / 2 / 3$, as well as any redundancy, in the context of Alkbh5 depletion in future studies. 
In conclusion, we provide mechanistic insight into epitranscriptional regulation of somatic cell reprogramming by elucidating the biphasic regulatory role of ALKBH5 in modulating reprogramming efficiency at the posttranscriptional level in a stage specific manner (Fig. 5).

\section{Materials and Methods}

\section{MEFs derivation}

All of Wild type (WT), Knockout (KO) Alkbh5 and homozygous floxed Alkbh5 MEFs $\left(A l k b h 5^{f f f)}\right.$ were derived from embryos at 13.5 d.p.c. Mice were housed and in Norwegian Transgenic Center (NTS). Briefly, pregnant mouse females were sacrificed on 13.5 or and embryos were dissected. The internal organs, head, and limbs were removed and used for genotyping. Then the remaining tissues were trypsinized using $0.25 \%$ trypsin for $30 \mathrm{~min}$ at $37^{\circ} \mathrm{C}$ with shaking to make single cell suspensions, then cells were pooled and plated in MEFs media until $80 \%$ confluence then trypsinized and stored in freezing solution (FBS+10\%DMSO) in liquid nitrogen for future use. MEFs were cultured and maintained in DMEM+10\% (tetracycline free FBS PAN-Biotech Catalog \# P30-2602TC ) till reaching to $70 \%$ to $80 \%$ confluence, then passaged at $1 \times 10^{5}$ cells per well of 6 -well plate.

$A l k b h 5^{f / f}$ MEFs were plated at $1 \times 10^{5}$ cells per well of 6-well plate O.N, next day the cells were transfected with KA1153_pPBCAG-MerCreMer-IN (Addgene Plasmid \#124183) together with PBase, and PB-GAC-Puro ( a kind gift from professor Hitoshi Niwa, Kumamoto University IMEG) using Lipofectamin 2000 (Invitrogen\# 11668019) or Fugene 6 (Promega \#E2691) for 5 hours, then the medium was changed. Next day, the cells were cultured with medium containing $2 \mu \mathrm{g} / \mathrm{ml}$ of Puromycin (Fisher Scientific \# A1113803) for 2 days, and then the cells were treated with $1 \mu \mathrm{M}$ of 4 Hydroxy Tamoxifen (4-OH Tam) (Merk\#H7904-5MG) for depletion of Alkbh5 at indicated time points . 


\section{Reprogramming}

For reprogramming MEFs at early passages were plated as single cells at $1 \times 10^{5}$ per well of 6 well plate or 5-6 x10\% $10 \mathrm{~cm}$ dish depending on the purpose of experiment. The cells were infected with equal ratio of the retroviruses expressing the four reprogramming factors (Oct4, Sox2, Klf4, and c-Myc), and incubated at $37^{\circ} \mathrm{C}$ for $8-12$ hours with $8 \mu \mathrm{g} / \mathrm{ml}$ of polybrene, then the medium changes next day. For either knockdown or overexpression experiments during reprogramming, the MEFs were plated at $1 \times 10^{5}$ per well of 6 well, and infected with lentivirus for 8 hours, them medium was changed, and next day the selectable markers were added for 2 days. If the cells were trypsinized at day 7 reprogramming, the reprogrammed cells cultured with feeder layer CF-1 MEFs Irradiated, P3 2M (AMS biotechnology \#GSC-6201G 2M or \#GSC-6101G 7M) and LIF ESGRO® Recombinant Mouse LIF Protein (1000 units/mL) (Millipore \# ESG1107). For induction of the transgene Stemolecule Doxycycline hyclate $10 \mathrm{mg}$ (Stemgent\#04-0016) was added at $1 \mu \mathrm{g} / \mathrm{ml}$ every 2 days.

\section{Retrovirus preparation}

Plate E cells were used for preparation of retrovirus (Cell bio labs \#RV-101) Plate E cells were plated at $1 \times 106$ cells per $10 \mathrm{~cm}$ dish in DMEM\%10FBS ( tetracycline free FBS PANBiotech Catalog \# P30-2602TC ) till reaching to $70 \%$ to $80 \%$ confluence, then cells were transfected with $9 \mu \mathrm{g}$ of each of pMXs-Oct4 (Addgene Plasmid \#13366), pMXs-Sox2 (Addgene Plasmid \#13367), pMXs-Klf4 (Addgene Plasmid \#13370), pMXs-c-Myc (Addgene Plasmid \#13375) per $10 \mathrm{~cm}$ dish using Fugene 6 (Catalog\# Promega\# E2691), and the medium was changed after 8 hours using (IMEDM+10\%FBS). Retroviral supernatant were harvested after 48 and 72 hours, spin down at 1200 r.p.m for 5 minutes at 4 c, and then used freshly or frozen in aliquots at $-80 \mathrm{c}$. The viral titer was estimated to produce up to $7-8 \%$ 
SSEA1 at day 7 of reprogramming or using GFP control estimated more than $85 \%$ infection efficiency by FACS .

\section{Lentivirus preparation}

LentiX 293T cells were used for preparation of lentivirus (Takahara Clontech \#632180). Lentix 293 T cells were plated at $1 \times 10^{6}$ cells per $10 \mathrm{~cm}$ dish in DMEM\%10FBS (tetracycline free FBS PANSera catalog ) till the cells reaching to $70 \%$ to $80 \%$ confluence. Then cells were transfected with PsPAx2 (Addgene Plasmid \#12260), and pMD2.G (Addgene Plasmid \#12259), and the vector encoding either shRNA for Knockdown or overexpression Alkbh5 or Alkbh5-HA tagged at c terminal for overexpression using Fugene 6, and the medium was changed after 8 hours using (IMEDM+10\%FBS). Retroviral supernatant were harvested after 48 and 72 hours, spin down at 1200 r.p.m for 5 minutes at $4 \mathrm{c}$, and then used freshly or frozen in concentrated form using ( aliquots at -80c. The viral titer was estimated to produce up to $7-8 \%$ SSEA1 at day 7 of reprogramming.

\section{Cell proliferation assay}

Mouse embryonic fibroblasts (MEFs) were plated at $1 \times 10^{4}$ per well of 24 well plate at quadruplicate. Then, at each indicated time point four wells were trypsinized and counted independently using (Life Technologies \#C10228 Countess ${ }^{\mathrm{TM}}$ Cell Counting Chamber Slides). Medium was replaced every 2 days and the data are presented as mean \pm SD for quadruplicate samples.

For reprogramming experiment, MEFs were plated at 1x105 cells per well of 6-well plate in triplicate, and infected with equal molar ratio of retroviral titer encoding Oct4, Sox2, Klf4, and c-Myc for 6 hours then medium changed, 8 hours after infection, cells were treated with either ethanol or $1 \mu \mathrm{M}$ of 4-hydroxy- Tamoxifen (4-OH-Tam) for depletion of Alkbh5. Cells 
were trypsinized at indicated time points and counted. Medium was replaced every 2 days and the data are presented as mean \pm SD for triplicate samples.

\section{Genotyping}

Cells of tissue biopsies has been suspended in lysis buffer (1M Tris-PH 8, 5M NaCl, 0.5M EDTA PH8, 10\% SDS) and freshly added (Proteinase K 20mg/ml) and incubated at 37c for 4hrs to O.N, then $300 \mu \mathrm{l}$ of $5 \mathrm{M} \mathrm{NaCl}$, then vortex , and incubated in ice for $10 \mathrm{~min}$, then spinning at low speed, remove the supernatant, and transfer to new tube , then add $650 \mu 1$ Isopropanol, vortex , and incubate at RT for $15 \mathrm{~min}$, then centrifuge at 150,000 r.p.m , then discard the supernatant, and dissolve the pellet in $200 \mu 1 \mathrm{TE}$ buffer, then incubate at $55 \mathrm{c}$ for $10 \mathrm{~min}$, then the DNA concentration is measured and 10-50 ng used per reaction.

\section{Cloning}

Both mAlkbh5 and mAlkbh5-HAtag were amplified from the cDNA using gateway forward and reverse primer using PrimeSTAR GXL DNA Polymerase (Takahara Clontech \# R050ATAK), and the PCR product was purified using QIAquick PCR Purification Kit (Qiagen \#28106), then shuttled to Gateway ${ }^{\mathrm{TM}}$ pDONR $^{\mathrm{TM}} 221$ Vector (Invitrogen\#12536017) using Gateway $^{\mathrm{TM}}$ BP Clonase ${ }^{\mathrm{TM}}$ II Enzyme mix (Invitrogen\#11789020), then transformed to One Shot $^{\mathrm{TM}}$ Stbl3 $^{\mathrm{TM}}$ Chemically Competent E. coli (Thermo Fisher \#C737303), and positive clones were screen by colony PCR, and restriction digestion, and positive colonies were sent for sequencing. The correct clone was used as entry clone and then the cloned gene was shuttled to destination vector pLX301 (Addgene Plasmid \#25895) For constitutive overexpression of either Alkbh5 or Alkbh5-HA, and pCW57.1 (Addgene Plasmid \#41393) for periodic overexpression of either Alkbh5 or Alkbh5-HA using LR clonase (Thermo \#11791020) based on manufacture protocol, and transformed to Stbl3 competent cells in case of Lentivirus destination vector, and positive clones were screen by colony PCR, and 
restriction digestion, and positive colonies were sent for sequencing, and confirmation, the positive colony was propagated and the plasmids were purified using Qiagen (Endotoxin free kit \#12362), and used for making the virus.

For shRNA cloning

Two short hairpins shRNA for targeting mAlkbh5 were annealed in annealing buffer by heating 10 minutes at 95C In PCR machine then cooling by gradual decreasing the temperature to $4 \mathrm{C}$ in 30 minutes, and then the annealed oligos were ligated using T4 DNA Ligase $(5 \mathrm{U} / \mu \mathrm{L}$ ) (Thermo Fisher Scientific \#EL0011) to either pLKO.1 puro (Addgene Plasmid \#8453) for constitutive knockdown or Tet-pLKO-puro (Addgene Plasmid \#21915) for periodic knockdown which was pre- linearized with AgeI-HF (NEB \# R3552L) and EcoRI-HF (NEB\#R3101S) restriction enzymes, then transformed to One Shot ${ }^{\mathrm{TM}} \mathrm{Stbl3}^{\mathrm{TM}}$ Chemically Competent E. coli (Thermo Fisher \#C737303), and several colonies were picked up and sent for sequencing. The positive clones were propagated and the plasmids were purified using Qiagen (Endotoxin free kit \#12362), and used for making the virus.

\section{qPCR}

TRIzol $^{\text {TM }}$ LS Reagent (Thermo scientific 10296010) was used for RNA extraction according to the manufacturer protocol, then the RNA was dissolved in UltraPure ${ }^{\mathrm{TM}} \mathrm{DNase} / \mathrm{RNase}-\mathrm{Free}$ Distilled Water (Thermo Scientific 10977049 ), then $1 \mu \mathrm{g}$ was used to make the cDNA using SuperScript $^{\mathrm{TM}}$ IV VILO ${ }^{\mathrm{TM}}$ Master Mix with ezDNase ${ }^{\mathrm{TM}}$ Enzyme (Thermo Scientific 11766050) based on manufacturer protocol. For Real time PCR, $2 \mu 1$ of cDNA was used per reaction using PowerUp ${ }^{\text {TM }}$ SYBR ${ }^{\text {TM }}$ Green Master Mix (Thermo Scientific A25777). The transcript level was normalized to the internal control. List of primers is attached in supplementary table 1

\section{RNA stability}


Cells were treated with $5 \mu \mathrm{g} / \mathrm{ml}$ of actinomycin D (Tocris \#1229). At indicted time point 3, 6, and 9 hours total RNA was extracted and DMSO treated cells was used as a control, and relative RNA expression was detected by qPCR

\section{m6A dot blot}

Total RNA was extracted from cells using TRIzol ${ }^{\mathrm{TM}}$ LS Reagent (Thermo scientific 10296010) or RNeasy Plus Mini Kit (Qiagen\# 74134). mRNA was isolated and purified using Dynabeads $^{\mathrm{TM}}$ mRNA Purification Kit (for mRNA purification from total RNA preps) (Invitrogen \# 61006) following the manufacturer's instructions. For m6A dot blot, mRNA was hybridized onto the Hybond-N+ membrane (GE Healthcare). After crosslinking spotted mRNA to membrane using Stratalinker 2400 UV Crosslinker, the membrane was blocked with 5\% skimmed milk for $1 \mathrm{~h}$, incubated with mouse anti-m6A antibody (1:1000, Millipore \# MABE1006) at $4^{\circ} \mathrm{C}$ overnight. Then the membrane was incubated with HRP-conjugated donkey anti-mouse IgG at room temperature for $1 \mathrm{~h}$. The membrane was photographed using the ECL imaging system (Bio-Rad). Finally, the membrane was stained with $0.02 \%$ methylene blue. Relative m6A level was quantified using ImageJ.

\section{m6A IP-qPCR}

Control and Alkbh5-HA overexpressed reprogrammed cells at day 12 of reprogrammed were harvested and mRNA was extracted from RNA as described previously. 1 to $2 \mu \mathrm{g}$ of mRNA was fragmented for 4 minutes at $70^{\circ} \mathrm{c}$ for 4 minutes, and then mRNA was precipitated and the pellet was dissolved in Ultrapure DNase/RNase free water, then incubated with pre conjugated m6A/protein G (Dynabeads ${ }^{\text {TM }}$ Protein G for Immunoprecipitation\#10003) beads in IP buffer, and incubated at $4 c$ for O.N. The mRNA was isolated from the beads using Trizol LS, and the RNA was used to make cDNA using SuperScript ${ }^{\mathrm{TM}}$ IV VILO ${ }^{\mathrm{TM}}$ Master Mix with ezDNase ${ }^{\mathrm{TM}}$ Enzyme (Thermo Scientific 11766050) based on manufacturer protocol 
. The m6A mRNA level was finally determined by real-time quantitative PCR relative to the input.

\section{Western blot}

Cells were washed twice with ice cold 1xPBS, and then scrapped and transferred to $1.5 \mathrm{ml}$ Eppendorf tube, then centrifuged, and supernatant was discarded, and the cells were lysed on RIPA lysis buffer (20mM Tris- $\mathrm{HCl} \mathrm{PH} 7.5,1 \mathrm{mM} \mathrm{MgCl} 2,500 \mathrm{mM} \mathrm{NaCl}, 20 \%$ glycerol , $0.5 \%$ NP-40, 1mM EDTA, 1mM EGTA) and freshly added 1x Halt Protease Inhibitor cocktail (100X) (Thermo Fisher \#87786), then incubated on ice for $30 \mathrm{~min}$, then centrifuged at maximum speed for 30 minutes, then the supernatant was transferred to new Eppendorf, and then the protein content was measured using Bradford protein assay (BSA) method, and then equal amounts of protein was lysed with 1x Bolt ${ }^{\mathrm{TM}}$ LDS Sample Buffer (Thermo Scientific B0008), and 1x Bolt ${ }^{\mathrm{TM}}$ Sample Reducing Agent(Thermo Scientific B0009) , and loaded on Bolt ready gel (4-12\%), and then the protein was transferred to PVDF or nitrocellulose Biorad pads using Trans-Blot Turbo Transfer System. Then the membrane was blocked using 5\% skimmed milk in 1xTBST buffer, and then incubated with the primary antibody O.N was agitation. Next day, the membrane was washed 3 times using 1xTBST buffer, then incubated with the secondary antibody for $1 \mathrm{hr}$ at RT, and then washed 3 times using 1xTBST buffer, and then the protein detected with Pierce ${ }^{\mathrm{TM}}$ ECL Western Blotting Substrate (Thermo Fisher 32209) or SuperSignal ${ }^{\mathrm{TM}}$ West Femto Maximum Sensitivity Substrate (Thermo Fisher 34094), using Biorad ChemiDoc XRS, and Precision Plus Protein $^{\text {TM }}$ Dual Color Standards (Bio-Rad\#161-0374) as a protein standard . Antibodies list is attached supplementary table 2 .

\section{Alkaline phosphatase staining}


Alkaline phosphatase staining has been already done using Leukocyte Alkaline Phosphatase

Kit (Sigma 85L3R), based on the manufacturer protocol as previously described (Khodeer and Era, 2017).

\section{Cell cycle analysis.}

The cells were trypsinized, harvested and collected, and washed 2 times with 1xPBS, and then suspended in $300 \mu 1$ ice cold 1xPBS, and then $700 \mu 1$ ice cold $100 \%$ ethanol and incubated at $4^{\circ} \mathrm{C}$ for at least 30 minutes. Then the cells centrifuged and supernatant was aspired and

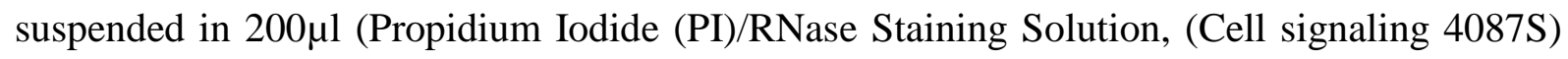
and incubated at RT for 30 minutes and then analyzed by FACS.

\section{Apoptosis.}

Detection of apoptotic cells was done by using FITC-Annexin V Apoptosis Detection Kit with 7-AAD (Biolegend\#640922). Briefly, The cells were collected, and washed 2 times with 1xPBS, and then suspended in $200 \mu 1 \mathrm{x}$ binding buffer, $1 \mu 1$ Annexin V- FITC, and 7AAD (1:200) and incubated at RT for 30 minutes in the dark. The cells were centrifuged and suspended in $300 \mu 11 \mathrm{x}$ binding buffer, and then analyzed by FACS.

\section{SSEA1 staining}

Cells at indicated time points were washed two times with 1xPBS, and trypsinized, precipitated, and counted. Then 1x106 cells were washed again with 1x Hanks buffer and stained with $5 \mu 1$ of Alexa Fluor ${ }^{\circledR} 647$ anti-mouse/human CD15 (SSEA-1) Antibody

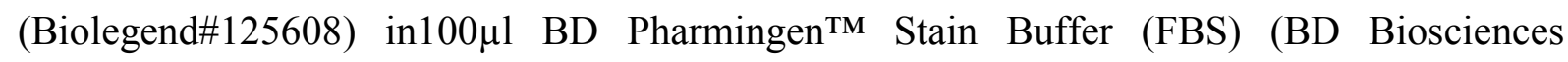
\#554656) for $30 \mathrm{~min}$ on Ice, and then cells were washed once with 1x Hanks buffer, then 7AAD (1:200), and SSEA1 positive fraction was analyzed using FACS BD Fortessa . 


\section{BrdU incorporation assay}

APC BrdU Flow Kit (BD Biosciences \#552598) was used according to the manufacturer's protocol. Briefly, cells were labeled by adding $10 \mu \mathrm{M}$ of BrdU to the culture medium. Treatment was done for 1 hour and then cells were fixed and permeabilized, and then treated with DNase for 1 hour at $37^{\circ} \mathrm{c}$, then stained with anti-BrdU APC for 20 minutes at RT, then resuspended in 7AAD and analyzed by FACS.

\section{Statistical Analysis}

All data were collected from at least three independent experiments. Data were analyzed using Student's $t$-test or one-way ANOVA followed using Graphpad software. Significance was presented as ${ }^{*} p<0.05,{ }^{* *} p<0.01$, and ${ }^{* * *} p<0.001$. Error bars represented mean \pm SD. 


\section{Figure legends}

Figure 1. Alkbh5 depletion impairs the somatic cell reprogramming efficiency.

(A) Relative expression of Alkbh5 during somatic cell reprogramming detected by qPCR. Mouse embryonic fibroblasts (MEFs) and mouse embryonic stem cells (ESCs) cultured in serum plus leukemia inhibitory factor LIF (S/L), were used as a negative and positive controls of the pluripotency, respectively. Data are normalized to the housekeeping gene glyceraldehyde-3-phosphate dehydrogenase (Gapdh).

(B) Immunoblot analysis of ALKBH5 protein level during reprogramming. Alpha-tubulin (ATUB) was used as loading control.

(C) Immunoblot analysis of ALKBH5 protein level in MEFs after lentiviral infection with either scrambled or two different shRNAs targeting Alkbh5. (A-TUB) was used as loading control.

(D) Experimental design showing the timing of Alkbh5 knockdown, onset of reprogramming, SSEA1 and ALP detection.

(E) Fraction of SSEA1 positive cells determined by FACS analysis after Alkbh5 knockdown during the early phase of reprogramming. MEFs are used as negative control.

(F) Reprogramming efficiency was measured by counting the number of ALP positive colonies.

(G) Fraction of SSEA1 positive cells determined by FACS for reprogrammed wild type (WT) and knockout (KO) Alkbh5 MEFs assessed at day 7 of reprogramming.

(H) Fraction of SSEA1 positive cells determined by FACS analysis of reprogrammed homozygous Floxed Alkbh5 $\left(A l k b h 5^{f / f}\right)$ treated with Ethanol as a control or $1 \mu \mathrm{M}$ of 4-hydroxy Tamoxifen (4-OH Tam) for depletion of Alkbh5 at either day 2or day 4. Data are shown as mean $\pm \mathrm{SD} ; \mathrm{n}=3, * \mathrm{P}<0.05, * * \mathrm{P}<0.01$, and $* * * \mathrm{P}<0.001$ mean $\pm \mathrm{SD}$ deviation of triplicate samples. 
Figure 2. Alkbh5 depletion induces G2/M cell cycle arrest, and impairs the MET process.

(A) Cell proliferation was assessed by FACS measured by BrdU incorporation on day 3 of reprogramming using either scrambled shRNA or two different shRNAs targeting Alkbh5.

(B) Quantification of mean percentage of each of the populations G1, S and G2/M from FACS data shown in figure $2 \mathrm{~A}$. The mean percentage of each population was written mean \pm S.D.

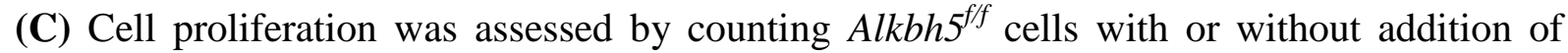
$1 \mu \mathrm{M}$ of 4-OH Tam for Alkbh5 depletion.

(D) Expression of mitotic checkpoint complex (MCC) factors as assessed by qPCR on day 3 of reprogramming using either scrambled shRNA or two different shRNAs targeting Alkbh5. The data are normalized to the housekeeping gene Gapdh.

(E) Immunoblot analysis of protein level for several cell cycle regulators on day 3 of reprogramming using either scrambled shRNA or two different shRNAs targeting Alkbh5, and (A-TUB) used as loading control.

(F) Expression of mesenchymal and epithelial genes as assessed by qPCR, on day 6 of reprogramming after infection either with scrambled shRNA or two different shRNAs targeting Alkbh5. The data are normalized to the housekeeping gene Gapdh.

(G) Immunoblot analysis of protein level for mesenchymal and epithelial markers on day 6 of reprogramming after infection either with scrambled shRNA or two different shRNAs targeting Alkbh5, and A-TUB was used as loading control. Data are shown as mean $\pm \mathrm{SD}$; $\mathrm{n}=3, * \mathrm{P}<0.05, * * \mathrm{P}<0.01$, and $* * * \mathrm{P}<0.001$. 
Figure 3. ALKBH5 overexpression enhances the reprogramming efficiency.

(A) Immunoblot analysis of ALKBH5 protein level after lentiviral infection of MEFs with empty vector or ALKBH5 or ALKBH5 tagged with HA (ALKBH5-HA), and A-TUB used as loading control.

(B) Fraction of SSEA1 positive cells determined by FACS analysis on day 14 of reprogramming after ALKBH5 overexpression.

(C) Reprogramming efficiency was measured by counting the number of ALP positive colonies on day 14 of reprogramming after ALKBH5 overexpression.

(D) Fraction of SSEA1 positive cells determined by FACS analysis on day 14 of reprogramming after temporal overexpression of ALKBH5-HA by $1 \mu \mathrm{g} / \mathrm{ml}$ doxycycline (Dox) from day 1 to day 7 , day 7 to day 14 or day 1 to day 14 . MEFs were used as a negative control. Data are shown as mean $\pm \mathrm{SD} ; \mathrm{n}=3, * \mathrm{P}<0.05, * * \mathrm{P}<0.01$, and $* * * \mathrm{P}<0.001$. 
Figure 4. ALKBH5 overexpression in the late phase of reprogramming stabilizes Nanog transcripts resulting in increased Nanog expression.

(A) Immunoblot analysis of ALKBH5 protein level after lentiviral infection of reprogrammed MEFs on day 12, with empty vector, ALKBH5 or ALKBH5-HA. $1 \mu \mathrm{g} / \mathrm{ml}$ of Dox was added on day 10, then cells were harvested on day 12, and A-TUB used as loading control.

(B) Endogenous expression of pluripotency factor (Oct4, Sox2, Klf4) as detected by qPCR, on day 12 of reprogramming using either empty vector, ALKBH5 or ALKBH5-HA. The data are normalized to the housekeeping gene Gapdh.

(C) Expression of pluripotency markers detected by qPCR, on day 12 of reprogramming using either empty vector, ALKBH5 or ALKBH5-HA. The data are normalized to the housekeeping gene Gapdh.

(D) $\mathrm{m}^{6} \mathrm{~A}$-IP qPCR data of Nanog on day 12 of reprogramming using empty vector, ALKBH5 or ALKBH5-HA overexpression. Data were shown as relative enrichment of $\mathrm{m}^{6} \mathrm{~A}$ normalized to percentage (\%) of input.

(E) Half life time of Nanog mRNA on day of reprogramming after ALKBH5-HA. Gapdh was used a negative control, and data of cells treated with $5 \mu \mathrm{M}$ Actinomycin D (ActD) were normalized to DMSO treated cells.

(F) Reprogramming efficiency was measured by counting the number of ALP positive colonies on day 14 of reprogramming. Data are shown as mean $\pm \mathrm{SD} ; \mathrm{n}=3, * \mathrm{P}<0.05$, $* * \mathrm{P}<0.01$, and $* * * \mathrm{P}<0.001$. 
Figure 5. Model showing the biphasic role of ALKBH5 in somatic cell reprogramming.

(A) Depletion of Alkbh5 specifically in the early phase of reprogramming decreases the reprogramming efficiency by reducing the expression of cyclin B1 and B2. (B) Depletion of Alkbh5 in the late phase of reprogramming has no impact on reprogramming efficiency. (C) Overexpression of ALKBH5 in the early phase of reprogramming does not affect the reprogramming efficiency. (D) Overexpression of ALKBH5 in the late phase enhances the reprogramming efficiency through increasing Nanog expression. 
Supplementary figure 1. Depletion of Alkbh5 in the early stage impairs the reprogramming efficiency.

(A) $\mathrm{m}^{6} \mathrm{~A}$ dot blot analysis of uninfected MEF or infected either with lentiviral encoding for scrambled shRNA, and two different shRNAs targeting Alkbh5 (upper panel). Methyl blue staining was used as control to eliminate the difference in loaded mRNA amount (lower panel).

(B) Immunoblot analysis of ALKBH5 protein level in WT and Alkbh5 KO MEFs. A-TUB was used as loading control.

(C) Fraction of SSEA1 positive cells determined by FACS in WT and Alkbh5 KO reprogrammed MEFs on day 14 of reprogramming.

(D) Immunoblot analysis of ALKBH5 protein level in MEFs infected with lentiviral encoding for scrambled shRNA, and two different shRNAs targeting Alkbh5. After selection with puromycin for 2 days, cells were treated with $1 \mu \mathrm{g} / \mathrm{ml}$ of Dox to induce the expression of shRNA . A-TUB was used as loading control.

(E) Fraction of SSEA1 positive cells determined by FACS in reprogrammed MEFs infected either by scrambled shRNA or shRNA targeting Alkbh5 with or without $1 \mu \mathrm{g} / \mathrm{ml}$ Dox treatment on day 7 of reprogramming. MEFs were used as a negative control.

(F) Fraction of SSEA1 positive cells determined by FACS in reprogrammed MEFs infected either by scrambled shRNA or shRNA targeting Alkbh5 with or without $1 \mu \mathrm{g} / \mathrm{ml}$ Dox treatment on day 14 of reprogramming. MEFs were used as a negative control.

(G) Experimental design for Alkbh5 depletion. Homozygous Alkbh5 $5^{f / f}$ MEFs were derived from mice at 13.5 days post coitum (d.p.c), before transfected with PB-GAG-Mer-Cre-Mer-, then selection with puromycin for 2 days, and treatment with $1 \mu \mathrm{M} 4-\mathrm{OH}$ Tam for induction of the Cre to remove Alkbh5.

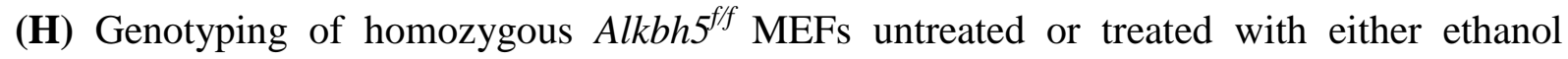
(negative control) or $1 \mu \mathrm{M} 4-\mathrm{OH}$ Tam for Alkbh5 removal. The band corresponds to the Neomycin (Neo) PCR amplicon of 515 base pairs (bps).

(I) ALKBH5 Immunoblot analysis of homozygous Alkbh $5^{f / f}$ MEFs untreated or treated with either ethanol (negative control) or $1 \mu \mathrm{M} 4-\mathrm{OH}$ Tam for Alkbh5 removal. 
(J) Fraction of SSEA1 positive cells determined by FACS in reprogrammed MEF on day 14

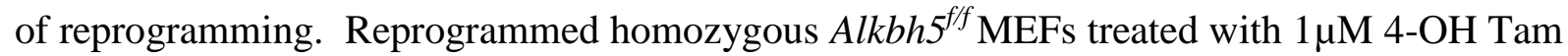
for Alkbh5 depletion at day 8 or day 10 of reprogramming.

(K) Reprogramming efficiency as assessed by counting the number of ALP positive colonies

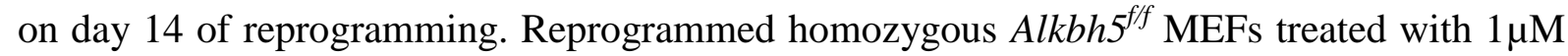
4-OH Tam for Alkbh5 at day 2, 4, 6, 8, 10 and 12 of reprogramming, and ethanol treatment was used as negative control. Data are shown as mean $\pm \mathrm{SD} ; \mathrm{n}=3, * \mathrm{P}<0.05, * * \mathrm{P}<0.01$, and $* * * \mathrm{P}<0.001$. 
Supplementary figure 2. Alkbh5 removal impairs the cell proliferation in either MEFs or reprogrammed MEF without increasing apoptosis.

(A) Fraction of apoptotic cells determined by FACS in reprogrammed MEFs uninfected or infected either by scrambled shRNA or two shRNAs targeting Alkbh5 was assessed at day 3 of reprogramming using double staining with Annexin V and 7AAD staining-.

(B) Analysis of cell apoptosis data determined by FACS in (Supplementary Fig. 2 A), each of 7AAD or Annexin V single positive (+ve) or negative (-ve), Annexin V/7AAD +ve or Annexin V/7AAD -ve.

(C) Only Annexin V/7AAD double positive population from (Supplementary Fig. 2 B) to clarify insignificance among reprogrammed MEFs; uninfected or infected either by scrambled shRNA or two shRNAs targeting Alkbh5 .N.S; Not significant.

(D) Expression of G1 cell cycle regulators as assessed by qPCR at day 3 of reprogramming in reprogrammed MEFs uninfected or infected either by scrambled shRNA or two shRNAs targeting Alkbh5 was estimated at day 3 of reprogramming. The data are normalized to the housekeeping gene Gapdh.

(E) Immunoblot analysis of protein level for several cell cycle regulators in either

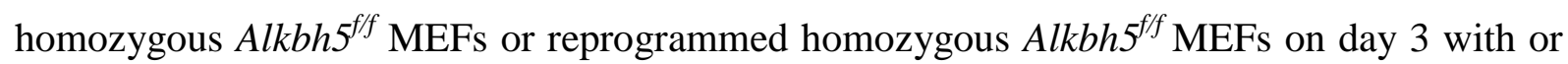
without treatment with $1 \mu \mathrm{M} 4-\mathrm{OH}$ Tam treatment to remove Alkbh5. A-TUB was used as loading control.

(F) Cell proliferation assay homozygous $A l k b h 5^{f / f}$ MEFs with or without treatment with $1 \mu \mathrm{M}$ 4-OH Tam treatment to remove Alkbh5 at different time points.

(G) Cell cycle analysis detected by PI staining and analyzed by FACS in uninfected MEFs or infected with scrambled shRNA or two different shRNAs targeting Alkbh5.

(H) Quantification of cell cycle phase G1, G2 and G2/M of data from Supplementary Fig. 2 G.

(I) Immunoblot analysis of protein level for several cell cycle regulators in homozygous $A l k b h 5^{f / f} \mathrm{MEFs}$ with or without treatment with $1 \mu \mathrm{M} 4-\mathrm{OH}$ Tam treatment to remove Alkbh5. A-TUB used as loading control. 
(J) Immunoblot analysis of protein level for both mesenchymal and epithelial markers in

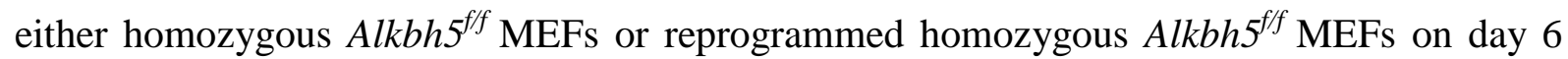
with or without treatment with $1 \mu \mathrm{M} 4-\mathrm{OH}$ Tam treatment to remove Alkbh5. A-TUB used as loading control.

(K) Phase contrast images of tracking morphological changes during reprogramming. Reprogrammed MEFs uninfected or infected either by scrambled shRNA or two shRNAs targeting Alkbh5 was estimated at day 2, 4, 6 and 14 of reprogramming. Data are shown as mean $\pm \mathrm{SD} ; \mathrm{n}=3, * \mathrm{P}<0.05, * * \mathrm{P}<0.01$, and $* * * \mathrm{P}<0.001$. 
Supplementary figure 4. ALKBH5 overexpression in the late phase of reprogramming enhances the reprogramming efficiency through increasing Nanog expression.

(A) Immunoblot analysis of ALKBH5 protein level after lentiviral infection of reprogrammed MEFs on day 12 with empty vector, ALKBH5 or ALKBH5-HA. $1 \mu \mathrm{g} / \mathrm{ml}$ of Dox was added on day 8 then cells were harvested on day10. A-TUB used as loading control.

(B) Endogenous expression of pluripotency factors (Oct4, Sox2, Klf4) as detected by qPCR, on day 10 of reprogramming using either empty vector, ALKBH5 or ALKBH5-HA. The data are normalized to the housekeeping gene Gapdh.

(C) Expression of pluripotency markers detected by qPCR, on day 12 of reprogramming using either empty vector, ALKBH5 or ALKBH5-HA. The data are normalized to the housekeeping gene Gapdh.

(D) Immunoblot of ALKBH5 in WT and KO Alkbh5 MEFs, and rescued KO MEFs infected with lentiviral ALKBH5 and ALKBH5-HA. A-TUB used as loading control.

(E) Phase contract image of Alkbh5 KO reprogrammed MEFs at day 14 of reprogramming.

(F) Phase contrast image of ALP stained of reprogrammed Alkbh5 KO MEFs, and rescued KO MEFs infected with either ALKBH5 or ALKBH5-HA at day 14. Data are shown as mean $\pm \mathrm{SD} ; \mathrm{n}=3, * \mathrm{P}<0.05, * * \mathrm{P}<0.01$, and $* * * \mathrm{P}<0.001$. 


\section{References}

1. Takahashi, K., et al., Induction of pluripotent stem cells from adult human fibroblasts by defined factors. cell, 2007. 131(5): p. 861-872.

2. Takahashi, K. and S. Yamanaka, Induction of pluripotent stem cells from mouse embryonic and adult fibroblast cultures by defined factors. cell, 2006. 126(4): p. 663676.

3. Chin, M.H., et al., Induced pluripotent stem cells and embryonic stem cells are distinguished by gene expression signatures. Cell stem cell, 2009. 5(1): p. 111-123.

4. Deng, J., et al., Targeted bisulfite sequencing reveals changes in DNA methylation associated with nuclear reprogramming. Nature biotechnology, 2009. 27(4): p. 353360.

5. Guenther, M.G., et al., Chromatin structure and gene expression programs of human embryonic and induced pluripotent stem cells. Cell stem cell, 2010. 7(2): p. 249-257.

6. Carey, B.W., et al., Reprogramming factor stoichiometry influences the epigenetic state and biological properties of induced pluripotent stem cells. Cell stem cell, 2011. 9(6): p. 588-598.

7. Onder, T.T. and G.Q. Daley, New lessons learned from disease modeling with induced pluripotent stem cells. Current opinion in genetics \& development, 2012. 22(5): p. 500-508.

8. Buganim, Y., et al., Single-cell expression analyses during cellular reprogramming reveal an early stochastic and a late hierarchic phase. Cell, 2012. 150(6): p. 12091222.

9. Samavarchi-Tehrani, P., et al., Functional genomics reveals a BMP-driven mesenchymal-to-epithelial transition in the initiation of somatic cell reprogramming. Cell stem cell, 2010. 7(1): p. 64-77.

10. Li, R., et al., A mesenchymal-to-epithelial transition initiates and is required for the nuclear reprogramming of mouse fibroblasts. Cell stem cell, 2010. 7(1): p. 51-63.

11. Stadtfeld, M. and K. Hochedlinger, Induced pluripotency: history, mechanisms, and applications. Genes \& development, 2010. 24(20): p. 2239-2263.

12. Banito, A., et al., Senescence impairs successful reprogramming to pluripotent stem cells. Genes \& development, 2009. 23(18): p. 2134-2139.

13. Kim, E.J., et al., BAK/BAX-mediated apoptosis is a Myc-induced Roadblock to reprogramming. Stem cell reports, 2018. 10(2): p. 331-338.

14. Kawamura, T., et al., Linking the p53 tumour suppressor pathway to somatic cell reprogramming. Nature, 2009. 460(7259): p. 1140-1144.

15. Li, H., et al., The Ink4/Arf locus is a barrier for iPS cell reprogramming. Nature, 2009. 460(7259): p. 1136-1139.

16. Hong, H., et al., Suppression of induced pluripotent stem cell generation by the p53p21 pathway. Nature, 2009. 460(7259): p. 1132-1135.

17. Utikal, J., et al., Immortalization eliminates a roadblock during cellular reprogramming into iPS cells. Nature, 2009. 460(7259): p. 1145-1148.

18. Zhang, C., J. Fu, and Y. Zhou, A review in research progress concerning $m 6 A$ methylation and immunoregulation. Frontiers in immunology, 2019. 10: p. 922.

19. Bokar, J., et al., Purification and cDNA cloning of the AdoMet-binding subunit of the human mRNA (N6-adenosine)-methyltransferase. Rna, 1997. 3(11): p. 1233-1247.

20. Schwartz, S., et al., Perturbation of $m 6 A$ writers reveals two distinct classes of $m R N A$ methylation at internal and 5' sites. Cell Rep 8: 284-296. 2014. 
bioRxiv preprint doi: https://doi org/10.1101/2021.05.10.443389; this version posted May 10, 2021. The copyright holder for this preprint

21. Liu, J., et al., A METTL3-METTL14 complex mediates mammalian nuclear RNA N 6adenosine methylation. Nature chemical biology, 2014. 10(2): p. 93.

22. Ping, X.-L., et al., Mammalian WTAP is a regulatory subunit of the RNA N6methyladenosine methyltransferase. Cell research, 2014. 24(2): p. 177-189.

23. Gerken, T., et al., The obesity-associated FTO gene encodes a 2-oxoglutaratedependent nucleic acid demethylase. Science, 2007. 318(5855): p. 1469-1472.

24. Zheng, G., et al., ALKBH5 is a mammalian RNA demethylase that impacts RNA metabolism and mouse fertility. Molecular cell, 2013. 49(1): p. 18-29.

25. Dominissini, D., et al., Topology of the human and mouse $m 6$ A RNA methylomes revealed by $m 6$ A-seq. Nature, 2012. 485(7397): p. 201-206.

26. Wang, X., et al., $N$ 6-methyladenosine-dependent regulation of messenger $R N A$ stability. Nature, 2014. 505(7481): p. 117-120.

27. Alarcón, C.R., et al., $N$ 6-methyladenosine marks primary microRNAs for processing. Nature, 2015. 519(7544): p. 482-485.

28. Tang, C., et al., ALKBH5-dependent m6A demethylation controls splicing and stability of long 3'-UTR mRNAs in male germ cells. Proceedings of the National Academy of Sciences, 2018. 115(2): p. E325-E333.

29. Song, H., et al., METTL3 and ALKBH5 oppositely regulate m6A modification of TFEB mRNA, which dictates the fate of hypoxia/reoxygenation-treated cardiomyocytes. Autophagy, 2019. 15(8): p. 1419-1437.

30. Chao, Y., J. Shang, and W. Ji, ALKBH5-m6A-FOXM1 signaling axis promotes proliferation and invasion of lung adenocarcinoma cells under intermittent hypoxia. Biochemical and biophysical research communications, 2020. 521(2): p. 499-506.

31. Zhang, C., et al., Hypoxia induces the breast cancer stem cell phenotype by HIFdependent and ALKBH5-mediated m6A-demethylation of NANOG mRNA. Proceedings of the National Academy of Sciences, 2016. 113(14): p. E2047-E2056.

32. Zhang, S., et al., m6A demethylase ALKBH5 maintains tumorigenicity of glioblastoma stem-like cells by sustaining FOXM1 expression and cell proliferation program. Cancer cell, 2017. 31(4): p. 591-606. e6.

33. Wang, J., et al., The biological function of $m 6 A$ demethylase ALKBH5 and its role in human disease. Cancer Cell International, 2020. 20(1): p. 1-7.

34. Batista, P.J., et al., m6A RNA modification controls cell fate transition in mammalian embryonic stem cells. Cell stem cell, 2014. 15(6): p. 707-719.

35. Geula, S., et al., $m 6 A m R N A$ methylation facilitates resolution of naïve pluripotency toward differentiation. Science, 2015. 347(6225): p. 1002-1006.

36. Hanna, J., et al., Direct cell reprogramming is a stochastic process amenable to acceleration. Nature, 2009. 462(7273): p. 595-601.

37. Gonzales, K.A.U., et al., Deterministic restriction on pluripotent state dissolution by cell-cycle pathways. Cell, 2015. 162(3): p. 564-579.

38. Wang, X.Q., et al., CDK1-PDK1-PI3K/Akt signaling pathway regulates embryonic and induced pluripotency. Cell Death \& Differentiation, 2017. 24(1): p. 38-48.

39. Huang, T., et al., FTO knockout causes chromosome instability and G2/M arrest in mouse GC-1 cells. Frontiers in genetics, 2019. 9: p. 732.

40. Pan, G. and J.A. Thomson, Nanog and transcriptional networks in embryonic stem cell pluripotency. Cell research, 2007. 17(1): p. 42-49.

41. Silva, J., et al., Nanog is the gateway to the pluripotent ground state. Cell, 2009. 138(4): p. 722-737.

42. Theunissen, T.W., et al., Nanog overcomes reprogramming barriers and induces pluripotency in minimal conditions. Current Biology, 2011. 21(1): p. 65-71. 
bioRxiv preprint doi: https://doi. org/10.1101/2021.05.10.443389; this version posted May 10, 2021. The copyright holder for this preprint

(which was not certified by peer review) is the author/funder, who has granted bioRxiv a license to display the preprint in perpetuity. It is made available under aCC-BY-NC-ND 4.0 International license.

43. Chen, X., et al., Integration of external signaling pathways with the core transcriptional network in embryonic stem cells. Cell, 2008. 133(6): p. 1106-1117.

44. Liu, J., et al., YTHDF2/3 are required for somatic reprogramming through different RNA deadenylation pathways. Cell Reports, 2020. 32(10): p. 108120.

45. Zaccara, S. and S.R. Jaffrey, A unified model for the function of YTHDF proteins in regulating $m 6 A$-modified $m R N A$. Cell, 2020. 181(7): p. 1582-1595. e18.

46. Lasman, L., et al., Context-dependent functional compensation between Ythdf m6A reader proteins. Genes \& development, 2020. 34(19-20): p. 1373-1391. 
Fig 1

A

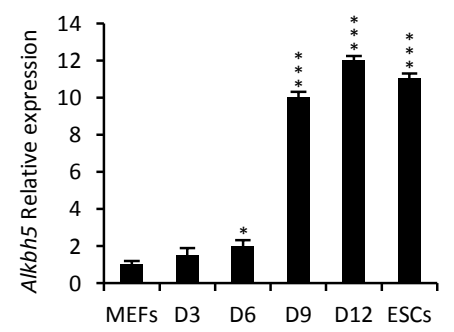

C

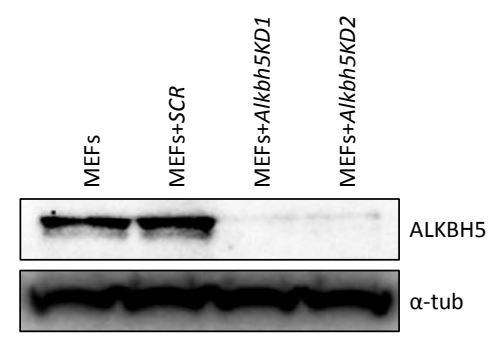

E

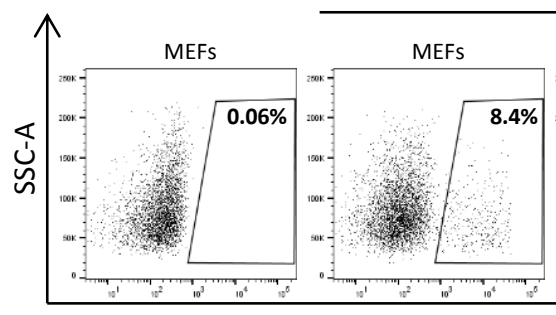

G

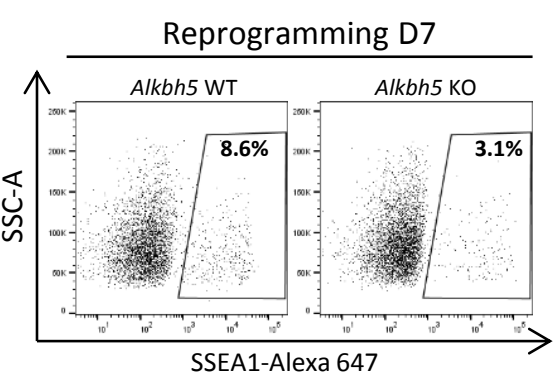

B

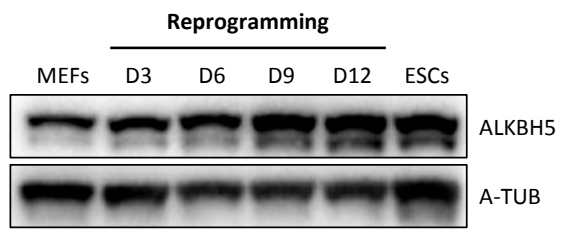

Reprogramming scheme

D

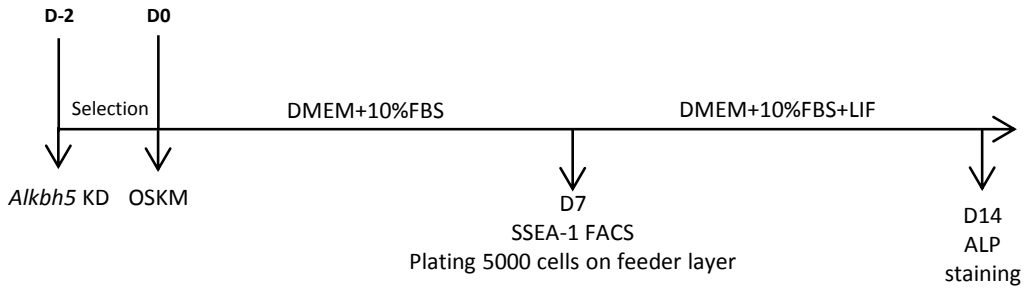

Reprogramming D7

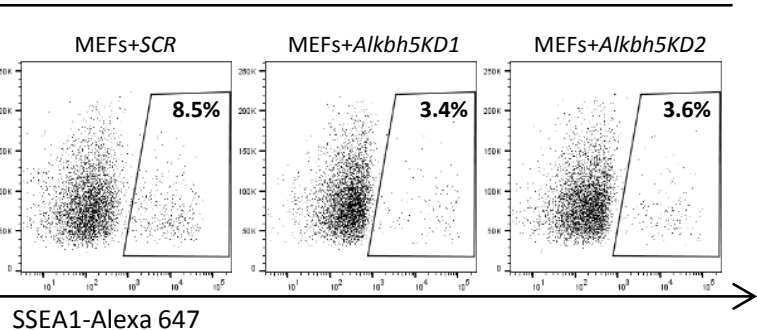

$\mathrm{H}$

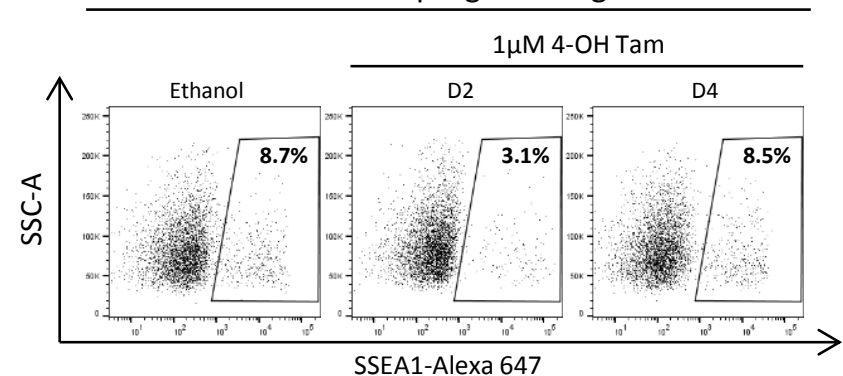

F

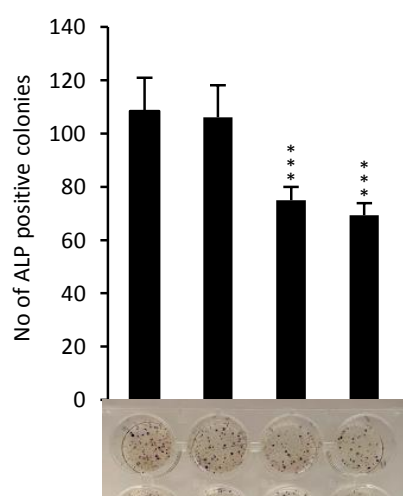


Fig 3

A

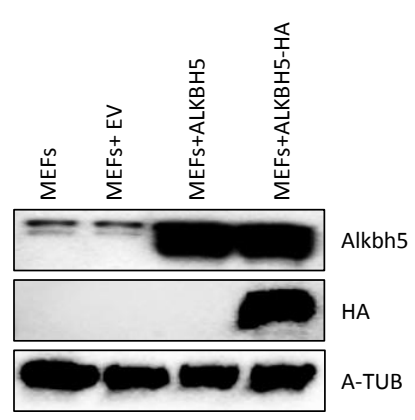

B

Reprogramming D14
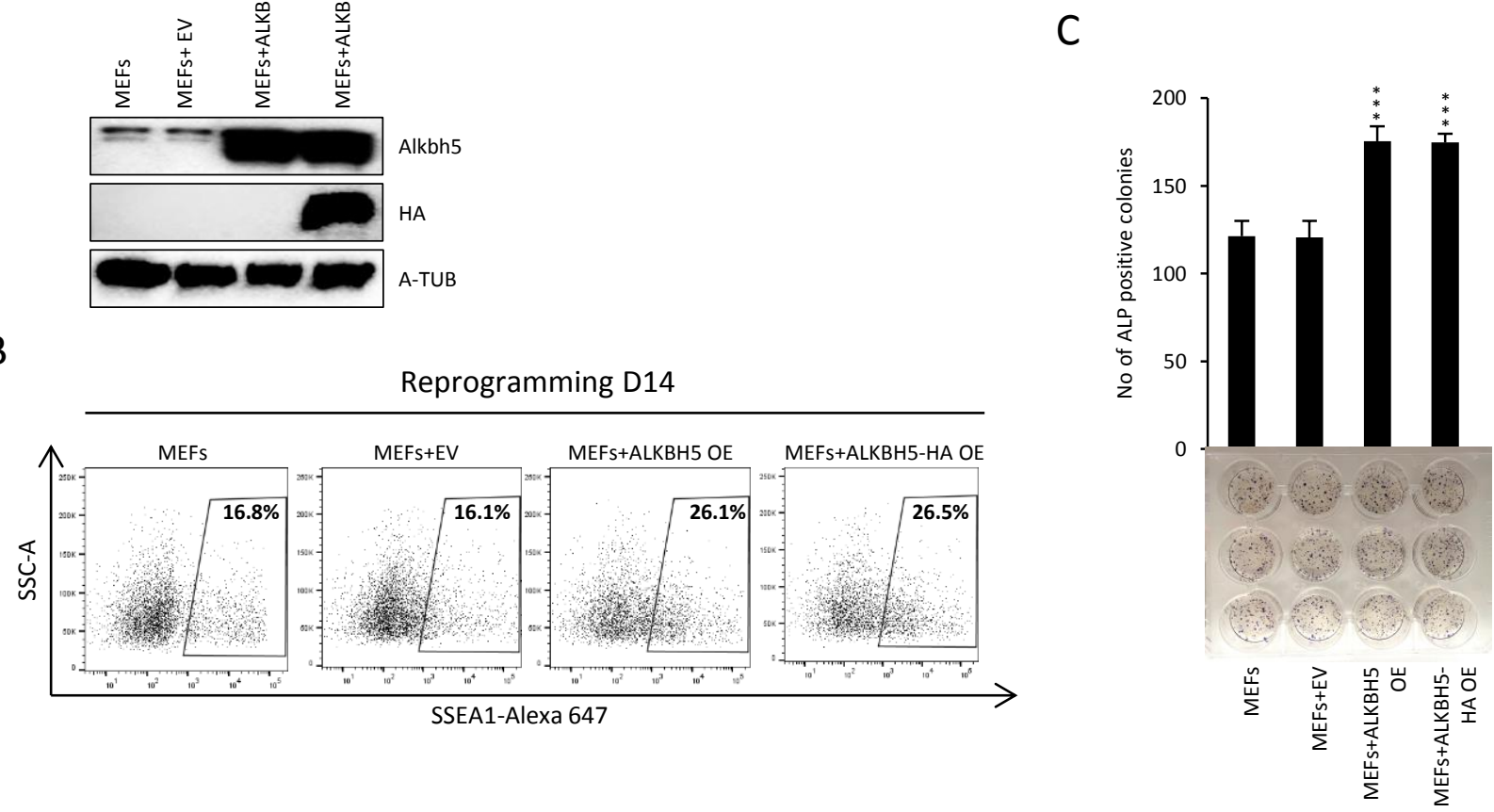

D

Reprogramming D14

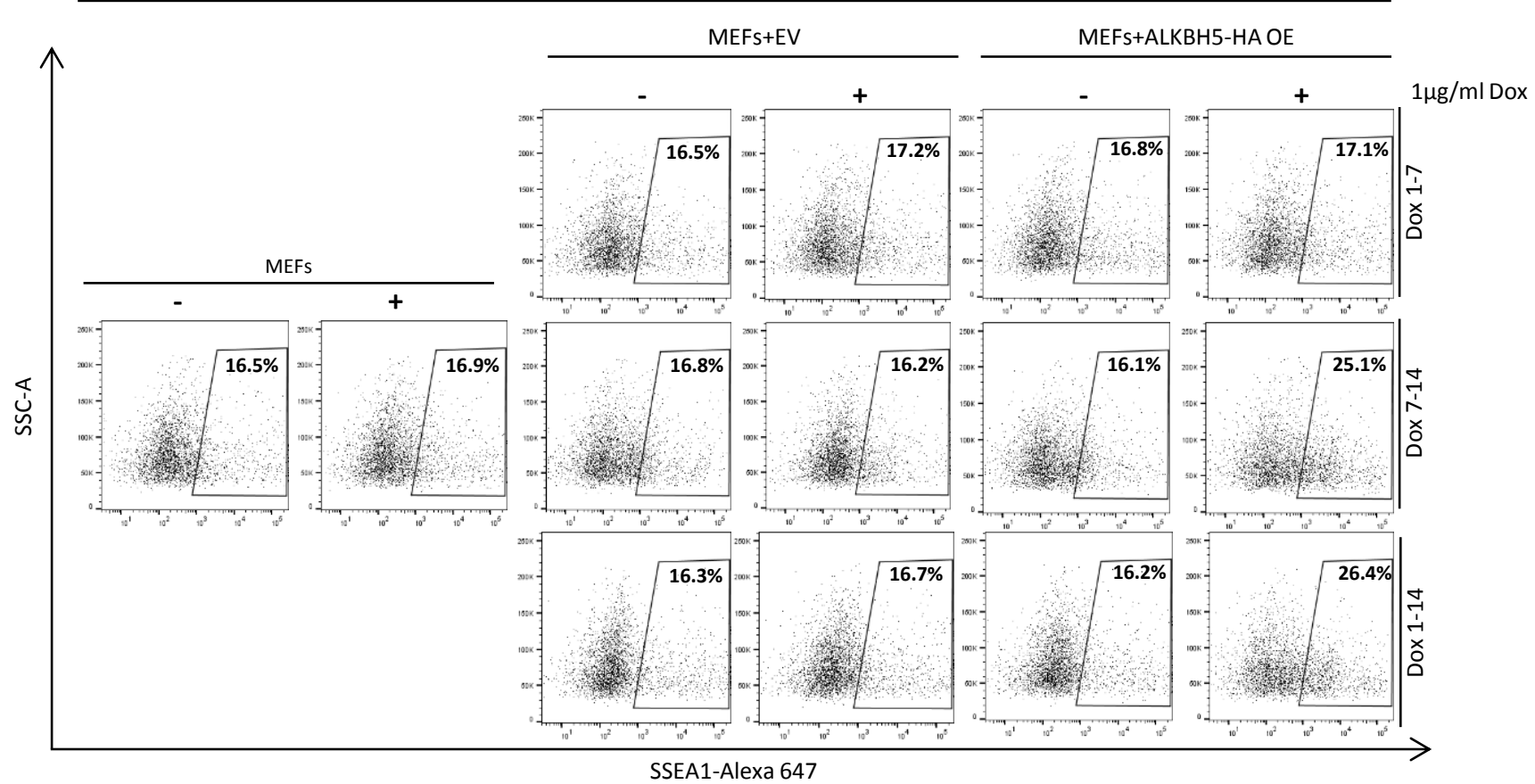


Fig 4

A

Reprogramming Day 12

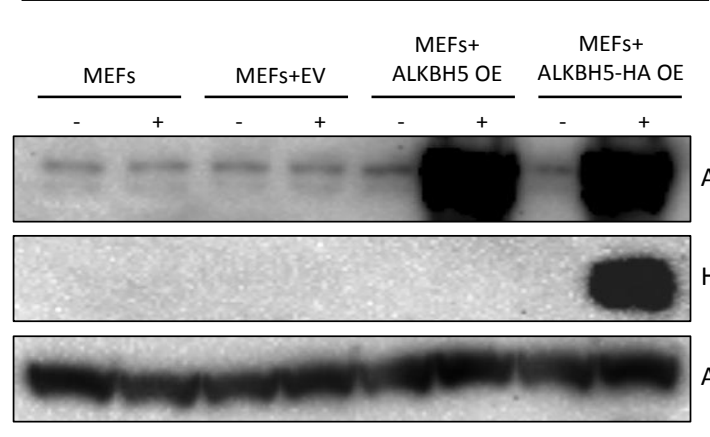

$1 \mu \mathrm{g} / \mathrm{ml}$ Dox

ALKBH5

HA

A-TUB

C

Reprogramming D12

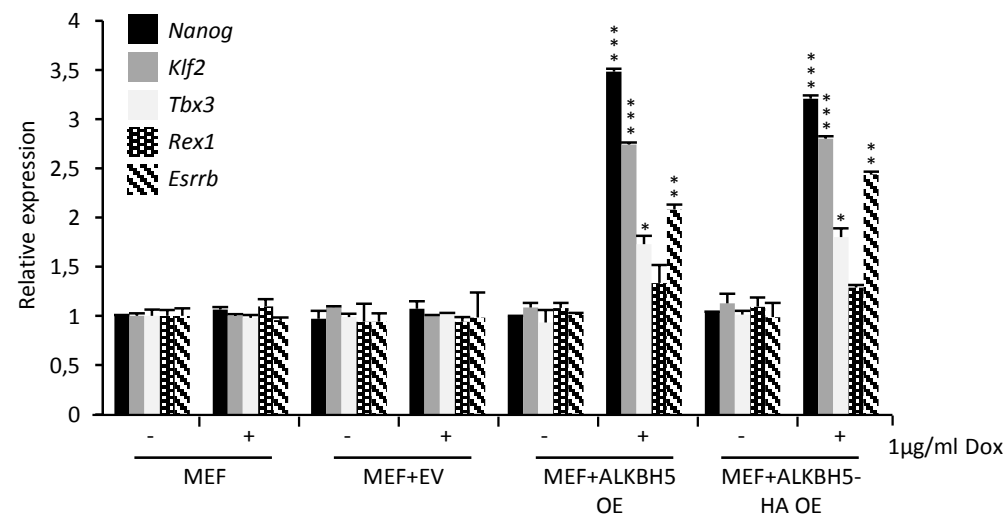

E

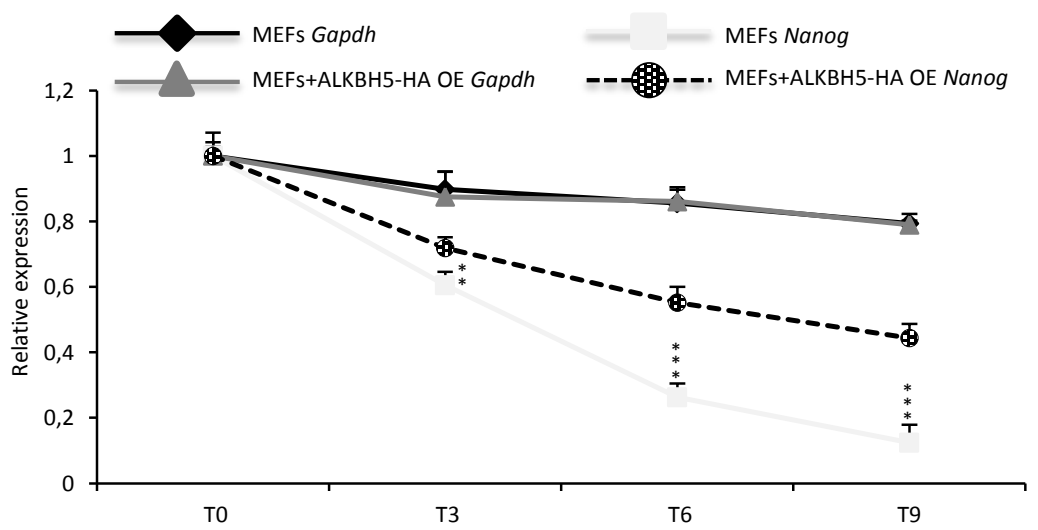

B

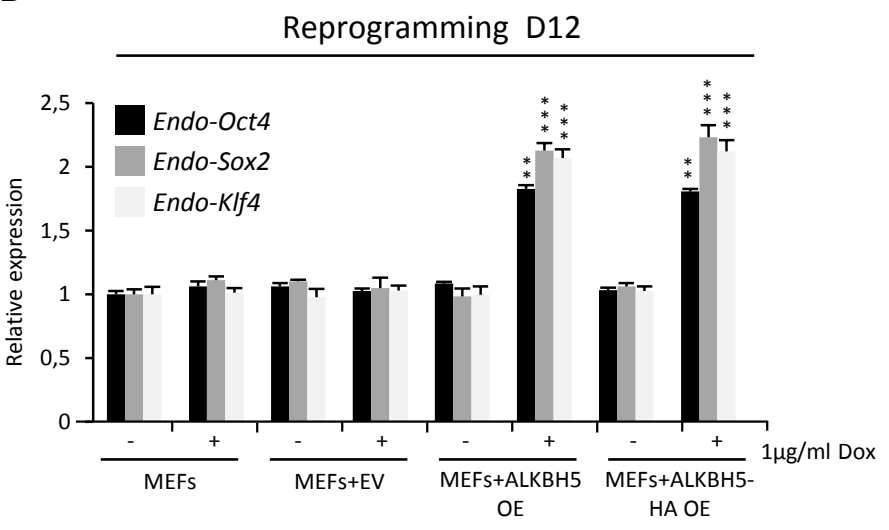

D

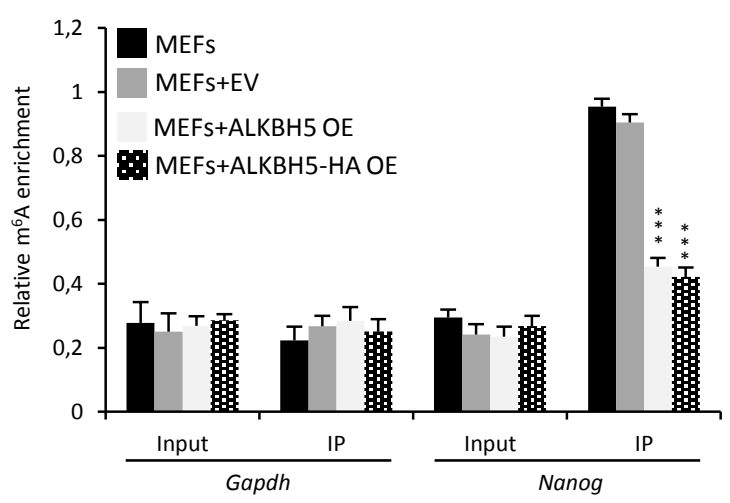

F 
Fig 5

A

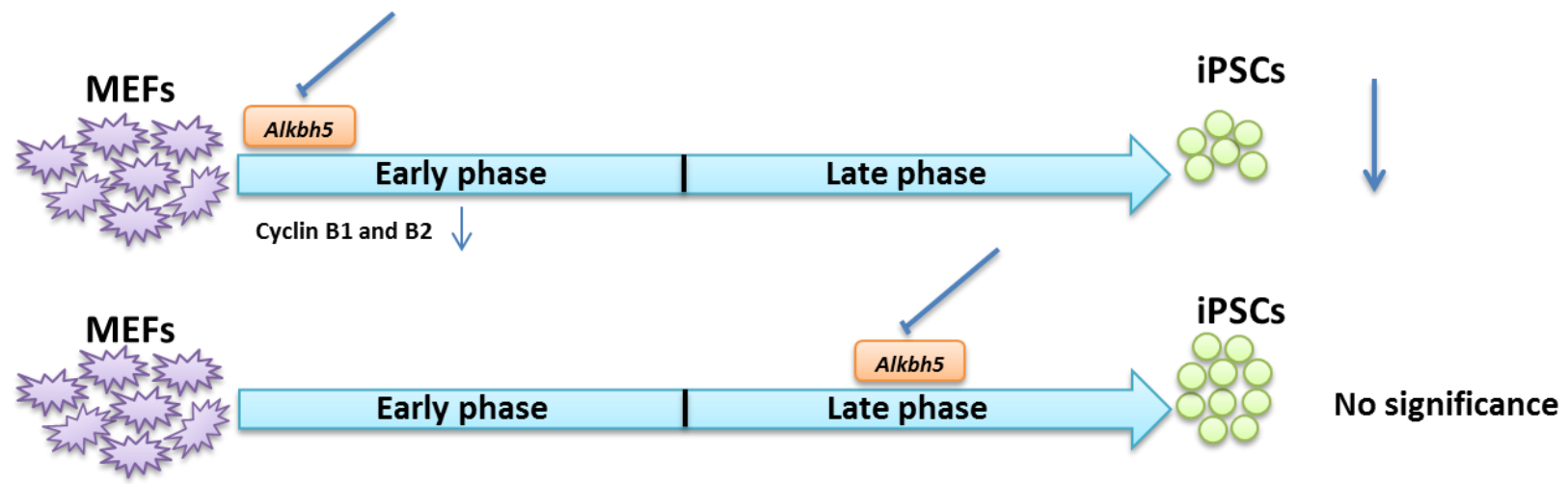

B

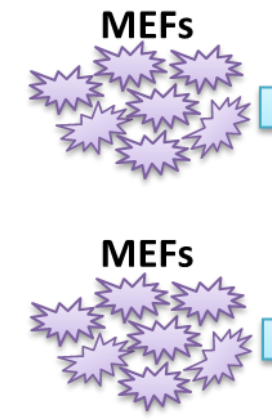

iPSCs

C

ALKBH5

\begin{tabular}{l|l} 
Early phase & Late phase
\end{tabular}

No significance

D

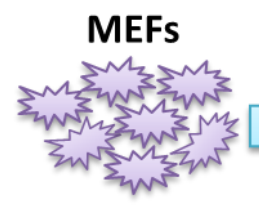

Early phase

ALKBH5

Late phase

Nanog $\uparrow$

iPSCs

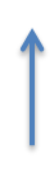

5 


\section{Supplementary Fig 1}

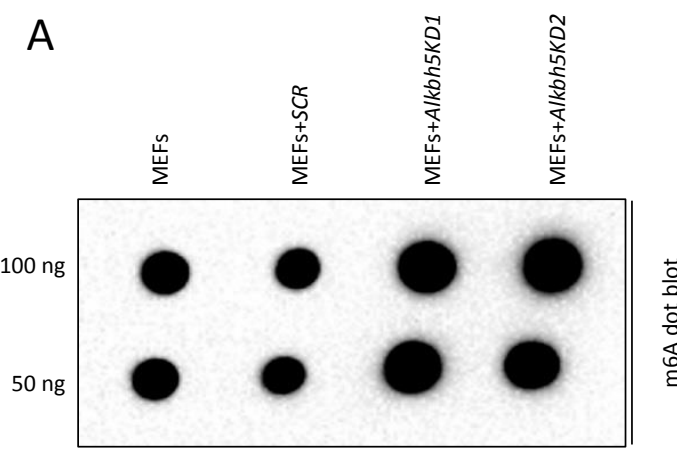

B
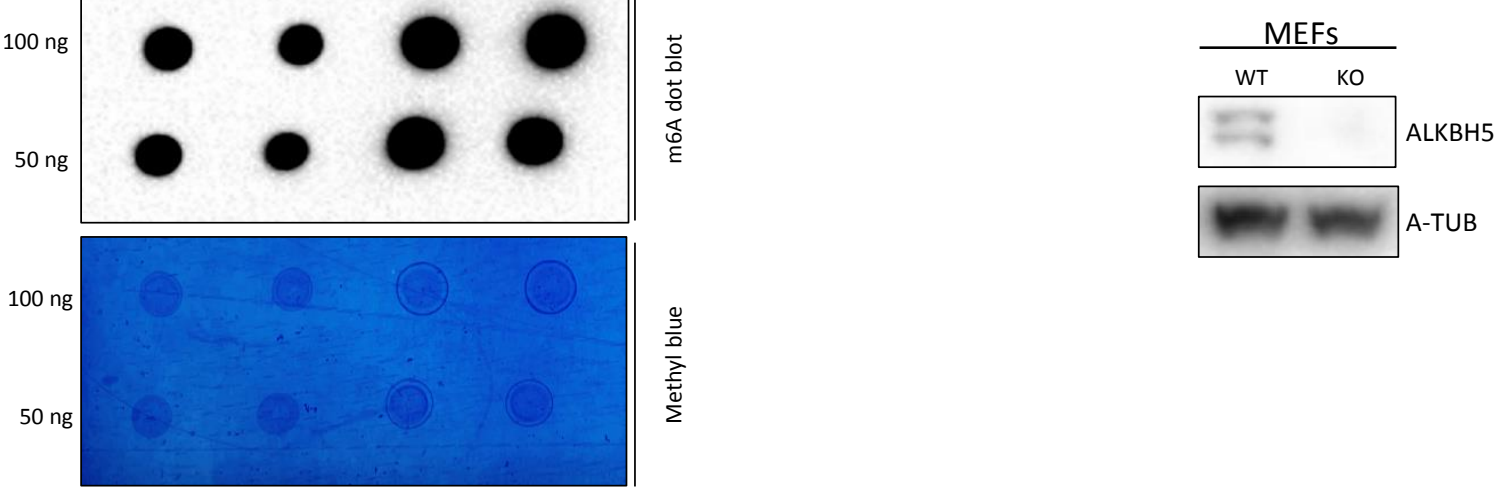

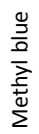

C

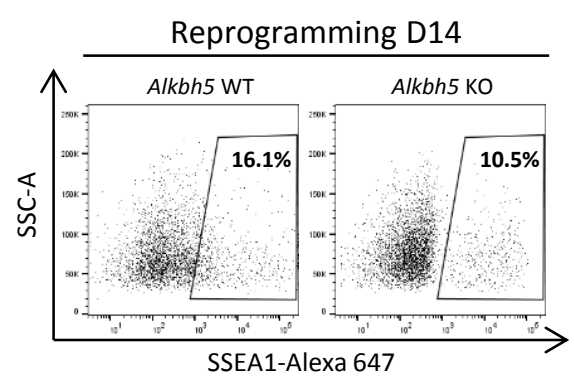

D

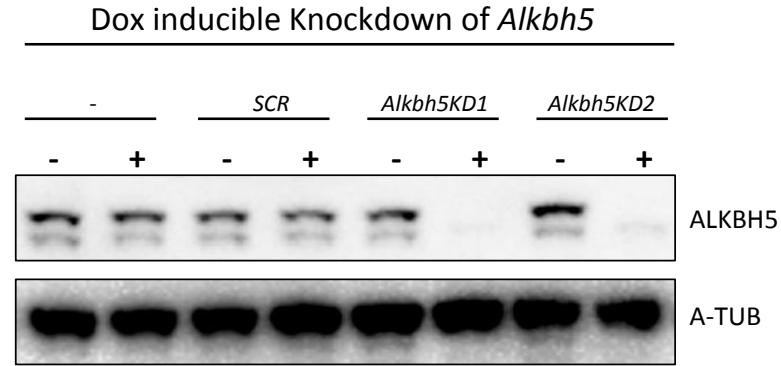

E

Reprogramming D7

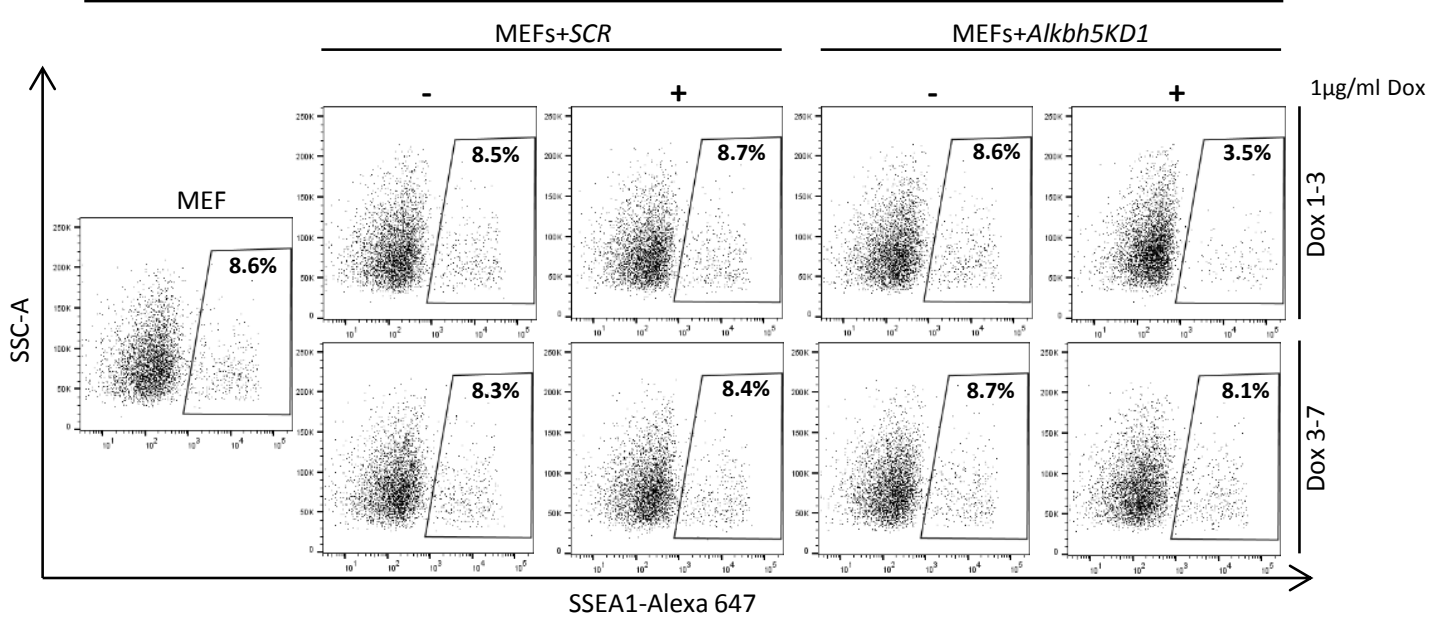




\section{Supplementary Fig 1}

F

Reprogramming D14
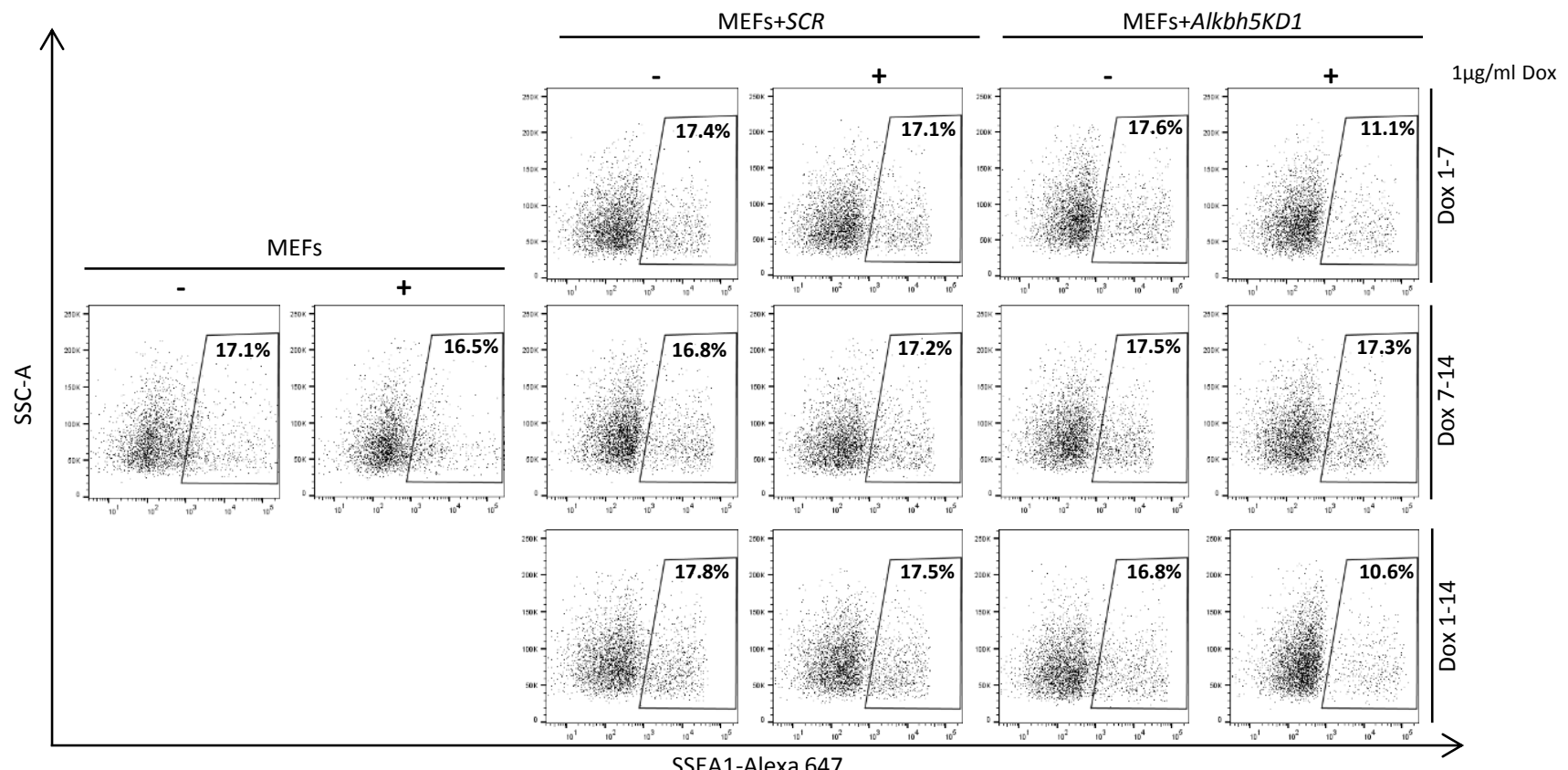

SSEA1-Alexa 647

G
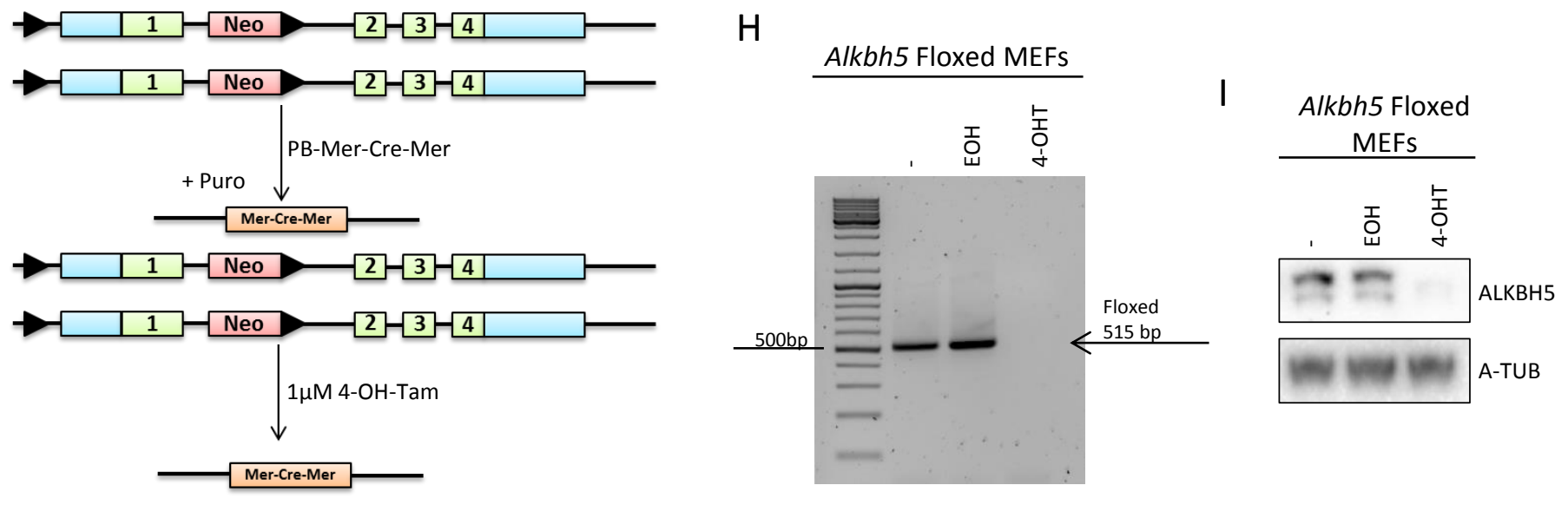


\section{Supplementary Fig 1}
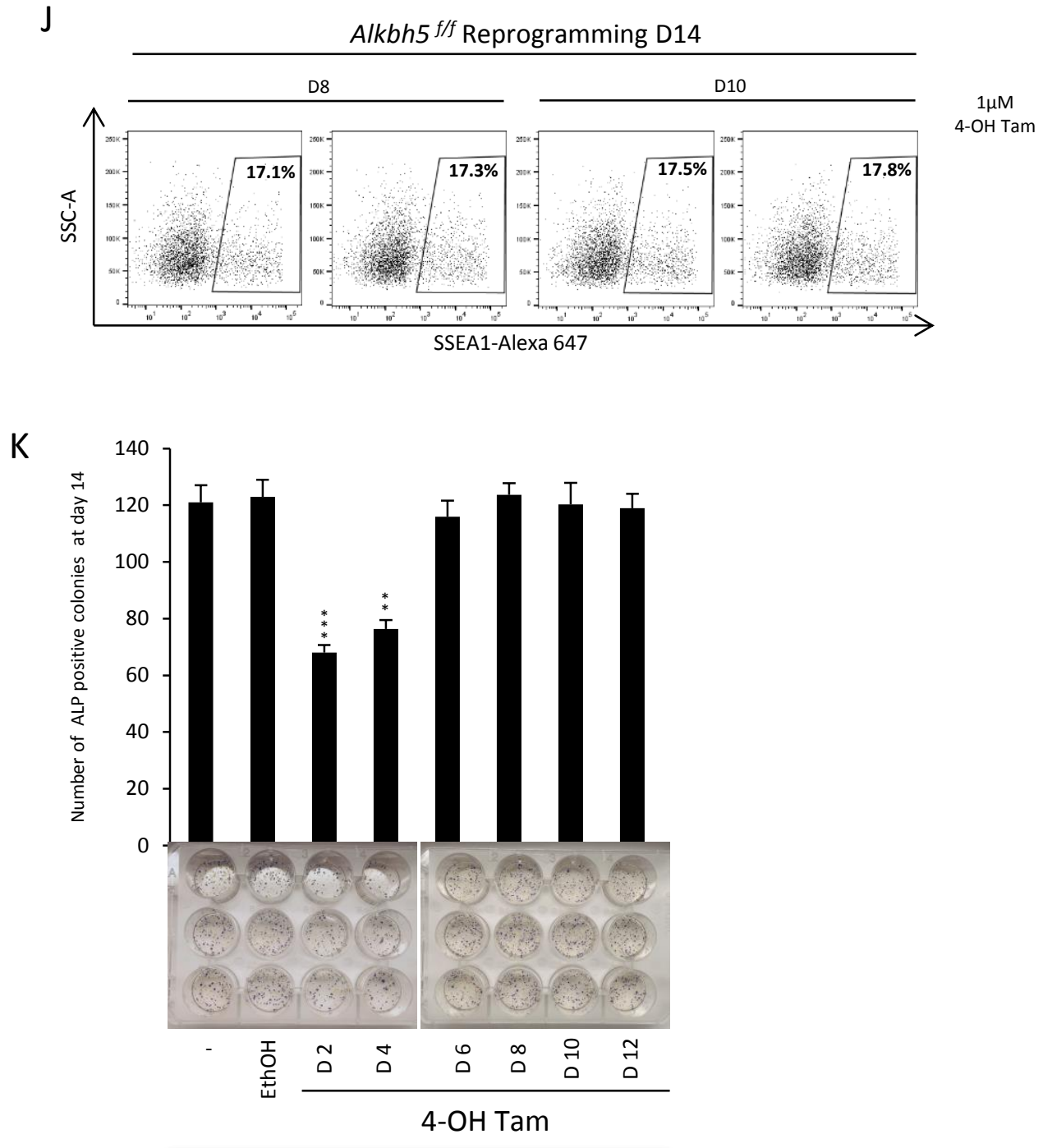

Alkbh5 f/f +Mer-Cre-Mer+ OSKM 


\section{Supplementary Fig 2}

A

Reprogramming D3

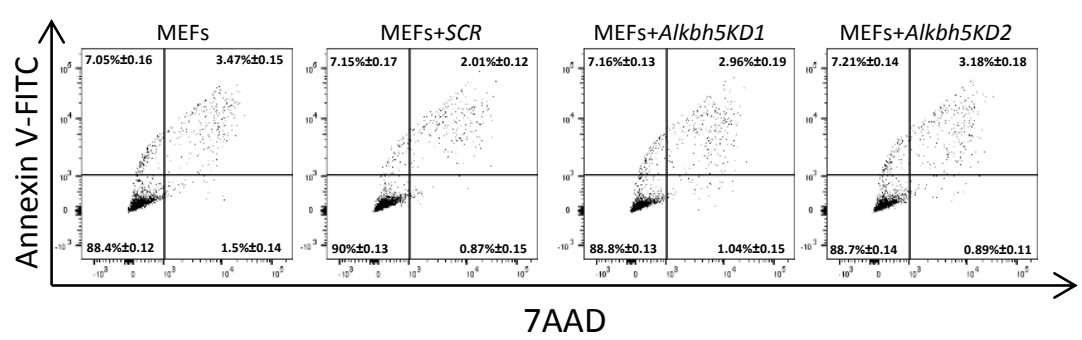

C

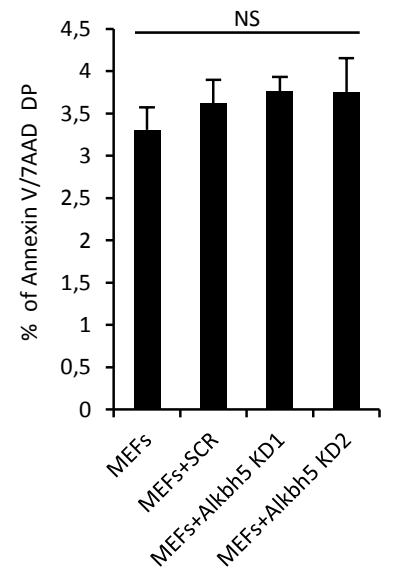

D

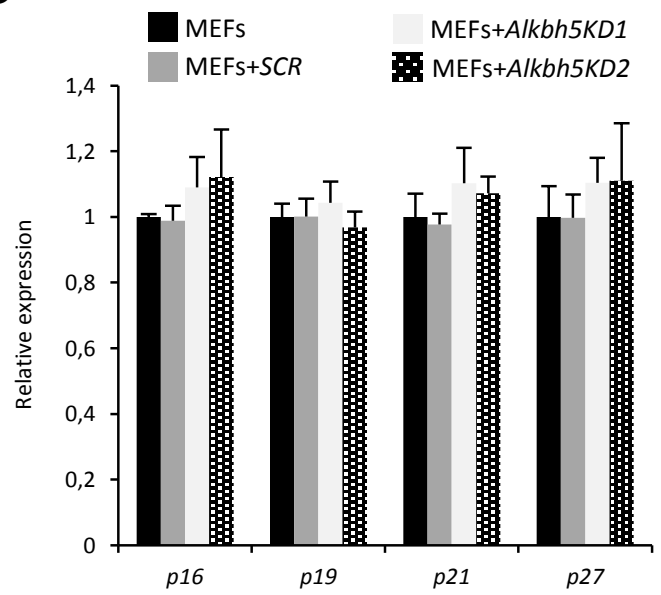

B

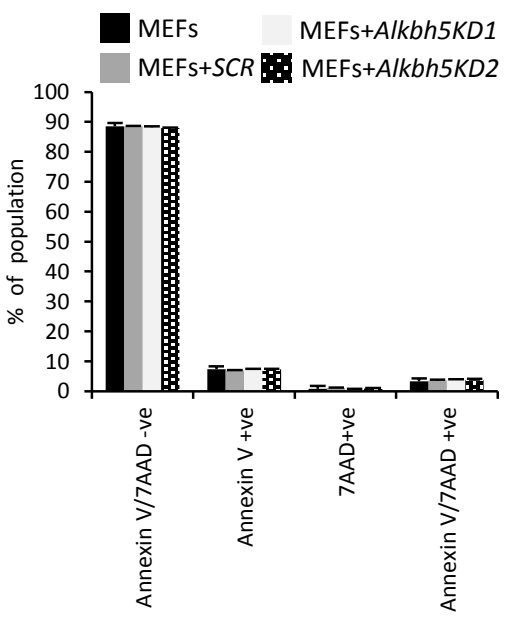

E

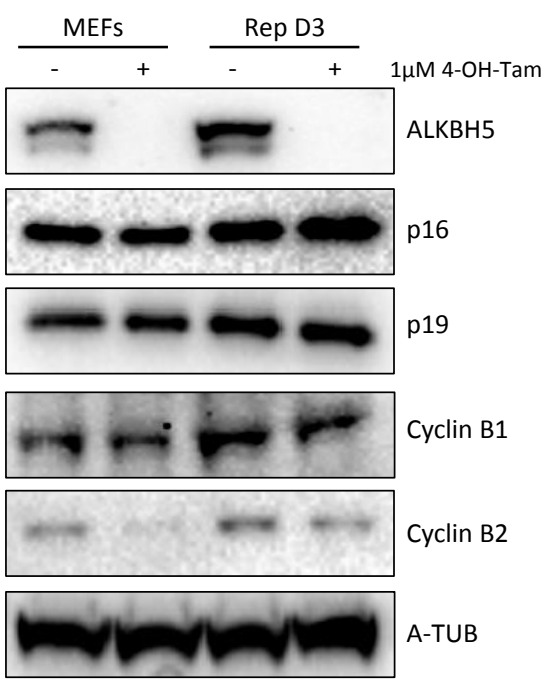




\section{Supplementary Fig 2}

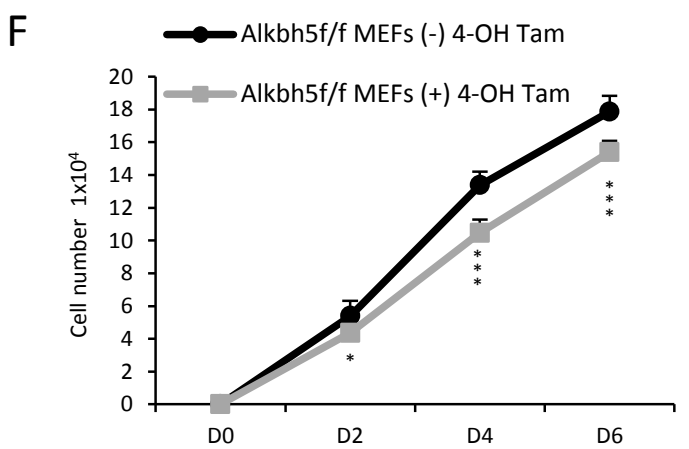

$\mathrm{H}$

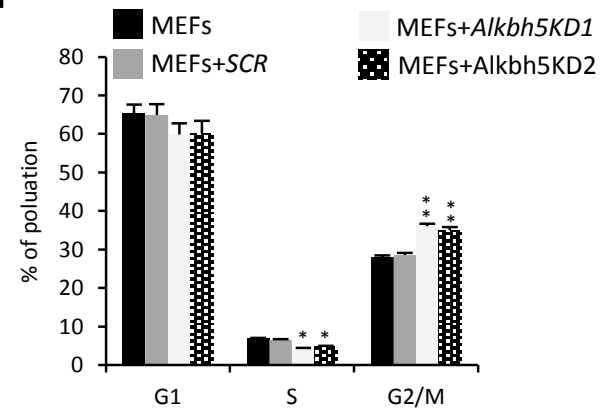

J
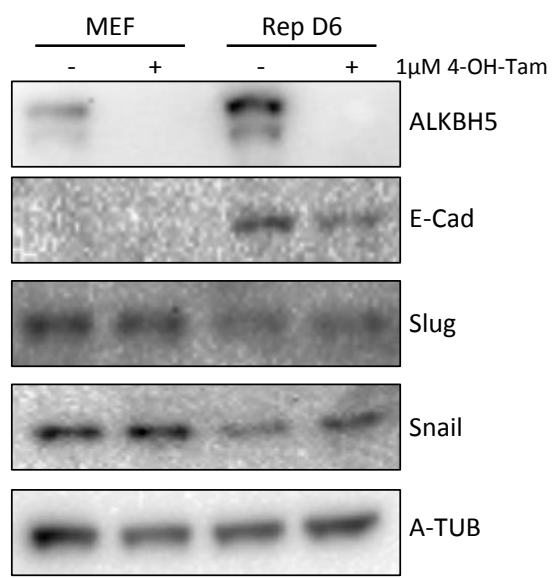

G

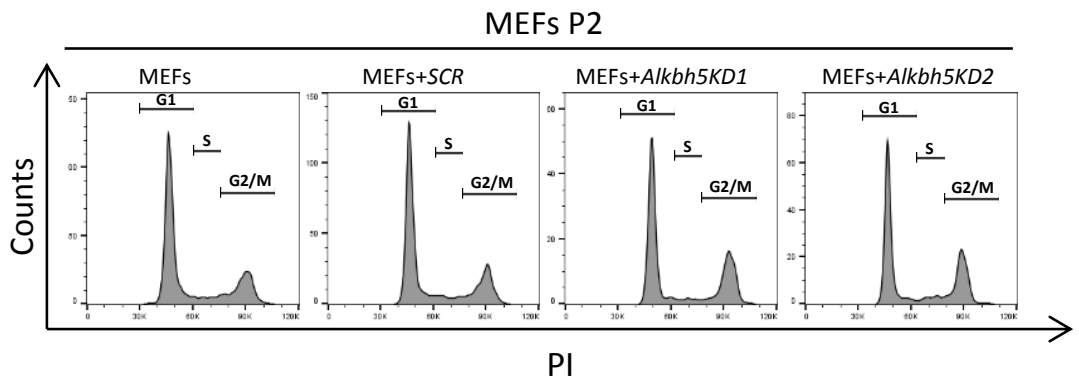

Alkbh5

$\begin{array}{ll}\text { Floxed MEFs } & \begin{array}{r}1 \mu \mathrm{M} \\ \text { 4-OH Tam }\end{array}\end{array}$

\begin{tabular}{|c|c|c|}
\hline$=$ & & ALKBH5 \\
\hline 70 & 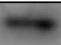 & p16 \\
\hline- & $\omega$ & p19 \\
\hline 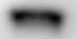 & $m$ & Cyclin B1 \\
\hline$\omega$ & & Cyclin B2 \\
\hline 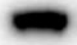 & $m$ & A-TUB \\
\hline
\end{tabular}




\section{Supplementary Fig 2}

Reprogramming

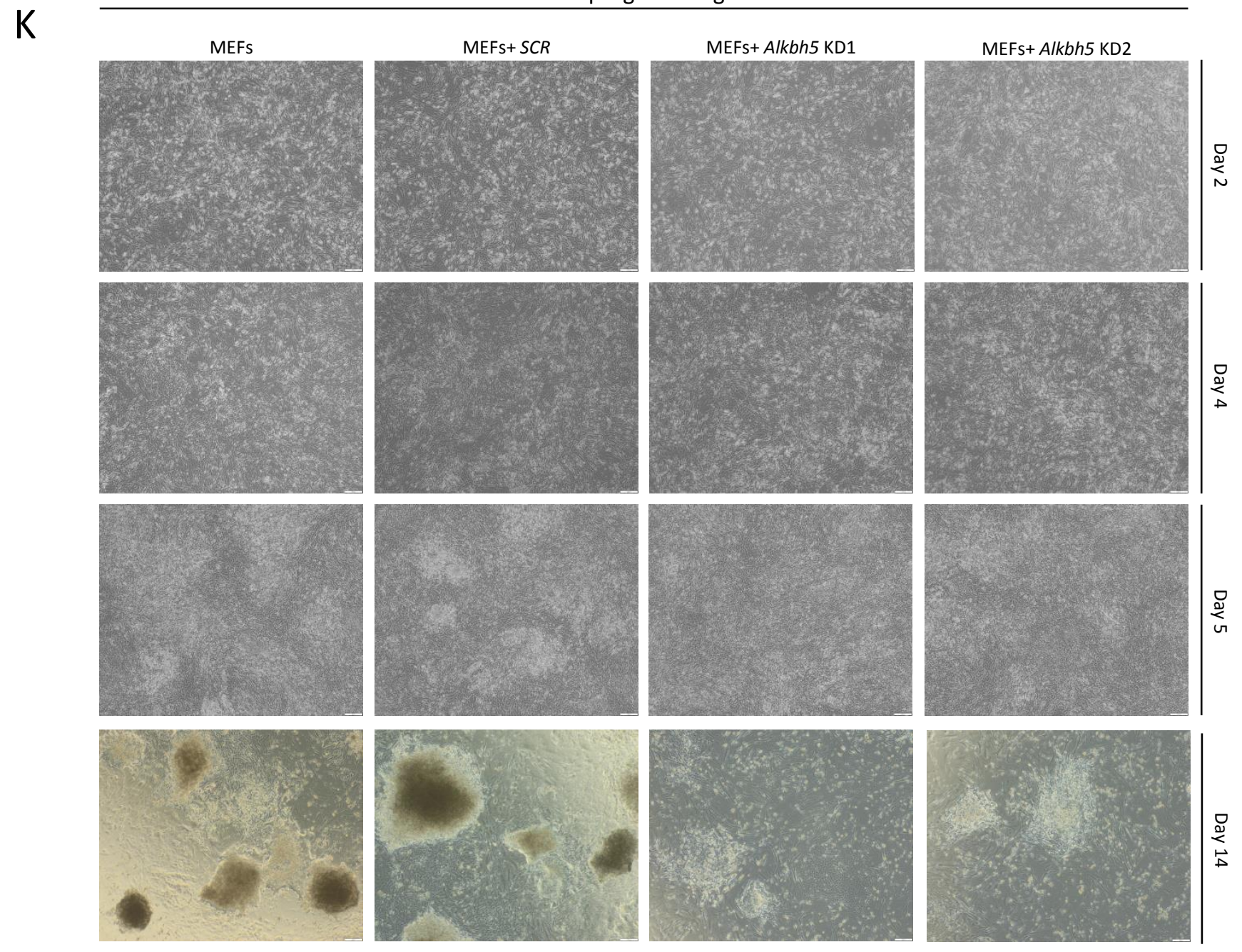




\section{Supplementary Fig 4}

A

Reprogramming Day 10

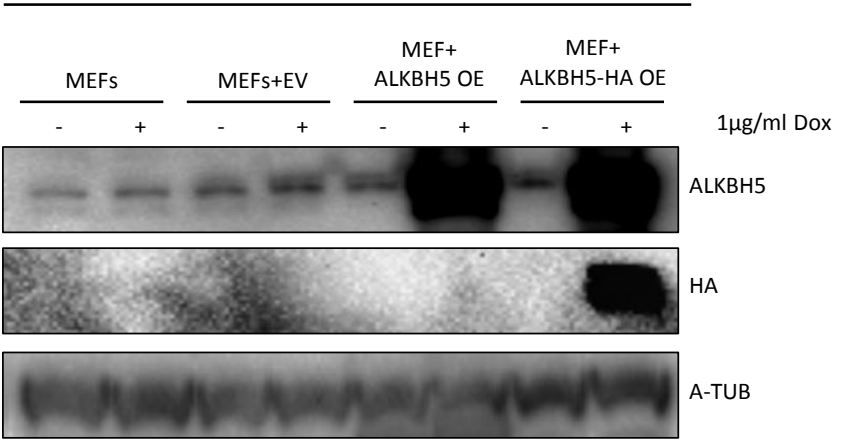

C

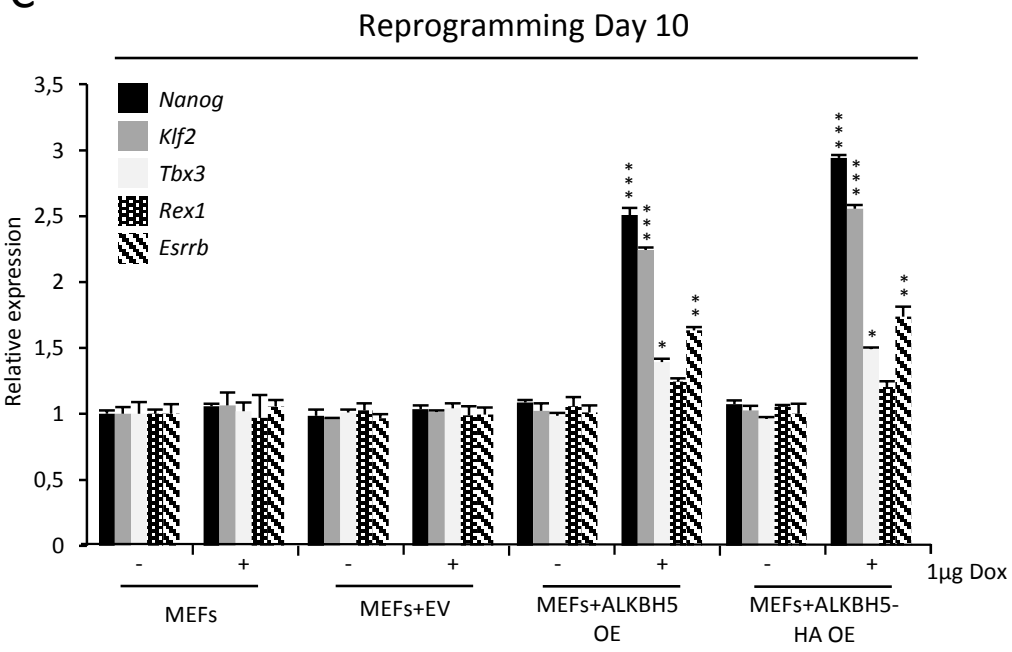

B Reprogramming Day 10

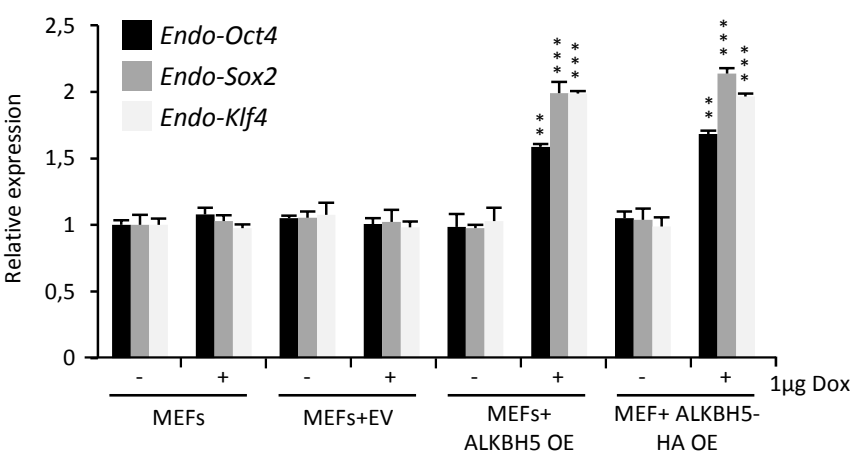

D

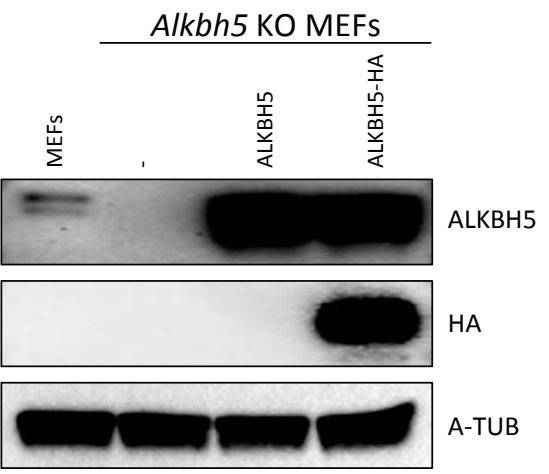

$E$

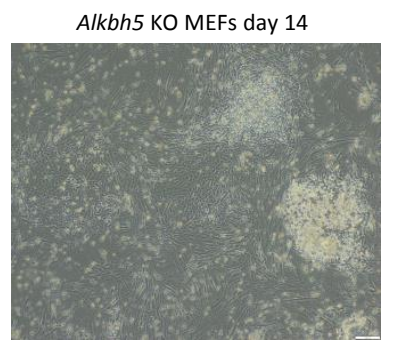

$\mathrm{F}$ ALP staining of reprogrammed cells on feeder layers at Day 14

Alkbh5 KO MEFs $\quad$ Alkbh5 KO MEFs+ALKBH5 Alkbh5 KO MEFs+ALKBH5-HA

